\title{
Lifting convex inequalities for bipartite bilinear programs
}

\author{
Xiaoyi $\mathrm{Gu}^{*} \quad$ Santanu S. Dey ${ }^{\dagger} \quad$ Jean-Philippe P. Richard ${ }^{\ddagger}$
}

June 25, 2021

\begin{abstract}
The goal of this paper is to derive new classes of valid convex inequalities for quadratically constrained quadratic programs (QCQPs) through the technique of lifting. Our first main result shows that, for sets described by one bipartite bilinear constraint together with bounds, it is always possible to sequentially lift a seed inequality that is valid for a restriction obtained by fixing variables to their bounds, when the lifting is accomplished using affine functions of the fixed variables. In this setting, sequential lifting involves solving a non-convex nonlinear optimization problem each time a variable is lifted, just as in Mixed Integer Linear Programming. To reduce the computational burden associated with this procedure, we develop a framework based on subadditive approximations of lifting functions that permits sequence-independent lifting of seed inequalities for separable bipartite bilinear sets. In particular, this framework permits the derivation of closed-form valid inequalities. We then study a separable bipartite bilinear set where the coefficients form a minimal cover with respect to the right-hand-side. For this set, we introduce a bilinear cover inequality, which is second-order cone representable. We argue that this bilinear cover inequality is strong by showing that it yields a constant-factor approximation of the convex hull of the original set. We study its lifting function and construct a two-slope subadditive upper bound. Using this subadditive approximation, we lift fixed variable pairs in closed-form, thus deriving a lifted bilinear cover inequality that is valid for general separable bipartite bilinear sets with box constraints.
\end{abstract}

\section{Introduction}

\subsection{Generating strong cutting planes through lifting}

Lifting is a technique that is used to derive or strengthen classes of cutting planes. It was first introduced to optimization in the context of mixed integer linear programming (MILP); see [46] for a review. The lifting process has two steps:

- Fixing and generation of a seed inequality: In the first step, the set $S$ of interest is restricted by fixing a subset of variables, say $x^{F}$, to specific values (typically to one of their bounds), say $\tilde{x}^{F}$. A valid inequality $h(x) \geq h_{0}$, which we call seed inequality, is then generated for the restriction $\left.S\right|_{x^{F}=\tilde{x}^{F}}$.

- Lifting the seed inequality: The seed inequality $h(x) \geq h_{0}$, when viewed with "zero coefficients" for the fixed variables $h(x)+0 \cdot x^{F} \geq h_{0}$ is typically not valid for the original set $S$. The task in the lifting step is to generate an inequality $h(x)+g\left(x^{F}\right) \geq h_{0}+g_{0}$, which (i) is valid for $S$ and (ii) satisfies $g\left(\tilde{x}^{F}\right)=g_{0}$. Under condition (ii), inequality $h(x)+g\left(x^{F}\right) \geq h_{0}+g_{0}$ reduces to inequality $h(x) \geq h_{0}$ when $x^{F}$ is set to $\tilde{x}^{F}$. The process of lifting is often accomplished by rotating or titling the seed inequality [27].

Though condition (ii) is not strictly necessary to impose, we require it in the remainder of the paper as otherwise $h(x)+g\left(x^{F}\right) \geq h_{0}+g_{0}$ is weak on the face $x^{F}=\tilde{x}^{F}$.

Lifting, as a technique for generating cutting-planes in MILP, has been extensively researched. Originally devised for node packing and knapsack sets [43, 44, 7, 35, 10, lifting was extended to general settings [54, 55, 31, 32, 47, 48, 4] and used to derive families of valid inequalities for many sets including [19, 38, 3, 1, 36, 22,

\footnotetext{
*xiaoyigu@gatech.edu, Georgia Institute of Technology

†santanu.dey@isye.gatech.edu, Georgia Institute of Technology

¡jrichar@umn.edu, University of Minnesota, Minneapolis
} 
56, 57, 58, 28. Many of the classes of cutting planes that have yielded significant computational gains can be obtained through lifting. This includes lifted cover inequalities [31, lifted tableaux cuts [22, 41], and even the Gomory mixed integer cut [26]; see [9, 50, 25, 21, 12, 11, 13, 6, 14, 24, 13, for papers related to lifting in the infinite group problem model. Similarly, mixing inequalities [33] can be viewed as an outcome of lifting [24].

Significantly fewer articles have focused on studying how lifting can be applied to nonlinear programs and mixed integer nonlinear programs. Exceptions include [51, which develops a general theory for lifting linear inequalities in nonlinear programming, [4] which applies lifting to derive the convex hull of a nonlinear set, 34] which studies lifting for the pooling problem, [5] which uses lifting for conic integer programs, and [20] which develops strong inequalities for mixed integer bilinear programs.

\subsection{Goal of this paper}

The goal of this paper is to derive new classes of valid convex inequalities for quadratically constrained quadratic programs (QCQPs) through the technique of lifting.

Generating valid inequalities for single row relaxations (together with bounds and integrality restrictions), i.e., for knapsack constraints, was the first, and arguably the most important step in the development of computationally useful cutting-planes in MILP. Motivated by this observation, various cutting-planes and convexification techniques for sets defined by a single non-convex quadratic constraint together with bounds have recently been investigated; see [20, 53, 2] for classes of valid inequalities for single constraint QCQPs and [23, 52] for convex hull results for such sets. The paper [45] studies a set similar to the one we study, albeit with integer variables. Further, 23] demonstrates that cuts obtained from one-row relaxations of QCQPs can be useful computationally. The paradigm of intersection cuts has also been explored to generate cuts for single-constraint QCQPs [40, 16]. Due to lack of space, we refrain from describing here the vast literature on convexification techniques for QCQPs and instead refer interested readers to [18, 52] and the references therein.

In this paper, we investigate the lifting of a convex seed inequality for a feasible region defined by a single (non-convex) quadratic constraint together with bound constraints. Apart from [5], we are not aware of any paper that attempts to study or employ lifting of convex nonlinear inequalities. To the best of our knowledge, this is the first study that derives lifted valid inequalities for general non-convex quadratic constraints with arbitrary number of variables. An extended abstract of this paper was accepted for publication in IPCO 2021 [30].

\subsection{Main contributions}

- Can we always lift? We present an example in two variables that illustrates that, even when a set is defined by a convex quadratic constraint, it might not always be possible to lift a linear seed inequality, valid for the restriction obtained by fixing a variable at lower bound, when we assume $g(\cdot)-g_{0}$ is an affine function of the fixed variable. Our main result, by contrast, establishes that there exists a large class of sets, described by a single bipartite bilinear constraint 23] together with bounds, for which it is always possible to lift when variables are fixed at their bounds. Note that any quadratic constraint can be relaxed to a bipartite bilinear constraint.

- Sequence-independent lifting. The lifting of a fixed variable requires the solution of a non-convex nonlinear optimization problem. When multiple variables must be lifted one at a time, this process (referred to as sequential lifting) can be computationally prohibitive. Further, the form of the lifted inequality obtained will differ depending on the order in which variables are lifted. For MILPs, it was shown in [55] that when the so-called lifting function is subadditive, lifting is far more computationally tractable in part because the form of the lifted inequality is independent of the order in which variables are lifted. We develop a similar general result for sequence-independent lifting of seed inequalities for separable bipartite bilinear constraints.

- Bilinear covering set and bilinear cover inequality. We next study a separable bipartite bilinear set whose coefficients form a minimal cover with respect to the right-hand-side. For this set, we derive a bilinear cover inequality. This second-order cone representable valid inequality yields a constant-factor approximation of the convex hull of the original set.

- Sequence-independent lifting of bilinear cover inequality. We construct a two-slope subadditive upper bound of the lifting function corresponding to the bilinear cover inequality. This function is reminiscent 
of the two-slope subadditive functions studied in the context of cutting-planes for the infinite group relaxation [29, 49, 37], although there is no apparent connection. Using this subadditive function, we lift fixed variable pairs in closed-form, thus describing a family of lifted bilinear cover inequalities, which are valid for general separable bipartite bilinear constraints.

Notation and organization of the paper Given a positive integer $n$, we denote the set $\{1, \ldots, n\}$ by $[n]$. Given a set $S \subseteq \mathbb{R}^{n}$ and $\theta>0$, we use $\theta \cdot S$ to denote the set $\{\theta x \mid x \in S\}$. We also use $\operatorname{conv}(S)$ to denote the convex hull of set $S$. The rest of the paper is organized as follows. In Section 2 we present our main results. In Section 3 we discuss some key directions for future research. Sections 4 10 give the proofs of the results described in Section 2 ,

\section{Main results}

Before we discuss our results, we first present two examples that illustrate how lifting can be performed for a set defined by a quadratic constraint and what challenges can arise during such procedure.

Example 1. Consider the set $S:=\left\{\left(x_{1}, x_{2}, x_{3}\right) \in[0,1]^{3} \mid x_{1} x_{2}+2 x_{1} x_{3} \geq 1\right\}$. First, we fix $x_{3}=0$ to obtain the restriction $\left.S\right|_{x_{3}=0}:=\left\{\left(x_{1}, x_{2}\right) \in[0,1]^{2} \mid x_{1} x_{2} \geq 1\right\}$. The seed inequality $\sqrt{x_{1} x_{2}} \geq 1$, is a valid convex inequality for $\left.S\right|_{x_{3}=0}$. We next show how it can be lifted into a valid inequality for $S$. Observe that, although valid for $\left.S\right|_{x_{3}=0}$, the seed inequality is not valid for $S$, since $\left(x_{1}, x_{2}, x_{3}\right)=(1,0,1 / 2)$ violates it while belonging to $S$. We therefore must introduce variable $x_{3}$ into the seed inequality so as to make it valid. In particular we seek $\alpha \in \mathbb{R}$ for which

$$
\sqrt{x_{1} x_{2}}+\alpha x_{3} \geq 1
$$

is valid for $S$. This question can be answered by solving the problem

$$
\begin{aligned}
\alpha^{*}:= & \sup \frac{1-\sqrt{x_{1} x_{2}}}{x_{3}} \\
& \text { s.t. } x_{1} x_{2}+2 x_{1} x_{3} \geq 1, x_{3} \in(0,1],\left(x_{1}, x_{2}\right) \in[0,1]^{2},
\end{aligned}
$$

where a key challenge is to first ascertain that the supremum is finite. When $\alpha^{*}$ is finite, it is clear that choosing any $\alpha \geq \alpha^{*}$ in (1) yields a valid inequality for $S$. Problem (2) can be analyzed using the following facts: (1) for any fixed value of $x_{3}$, we can always assume that an extreme point is the optimal solution, as the objective is to maximize a convex function, and (2) the extreme points of the set where $x_{3}$ is fixed to a value within its bounds are well-understood [52]. This suggests that one can inspect all different values of $x_{3}$ to establish that the supremum is finite. We illustrate these calculations next.

Observe that $\alpha^{*}$ can be obtained by computing the supremum $\alpha_{1}^{*}$ of (2) for $x_{3} \in[1 / 2,1]$ and then computing the supremum $\alpha_{2}^{*}$ of [2) for $x_{3} \in(0,1 / 2]$. When $x_{3} \in[1 / 2,1]$, one optimal solution is $x_{1}=\frac{1}{2 x_{3}}$ and $x_{2}=0$, thus $\alpha_{1}^{*}=\sup _{x_{3} \in[1 / 2,1]} \frac{1}{x_{3}}=2$. When $x_{3} \in(0,1 / 2]$, one optimal solution is $x_{1}=1$ and $x_{2}=1-2 x_{3}$, thus

$$
\alpha_{2}^{*}=\sup _{x_{3} \in(0,1 / 2]} \frac{1-\sqrt{1-2 x_{3}}}{x_{3}}=\sup _{x_{3} \in(0,1 / 2]} \frac{2}{1+\sqrt{1-2 x_{3}}}=2 .
$$

Choosing any $\alpha \geq \alpha^{*}=\max \left\{\alpha_{1}^{*}, \alpha_{2}^{*}\right\}=2$ yields a valid inequality for $S$. The strongest such valid inequality is $\sqrt{x_{1} x_{2}}+2 x_{3} \geq 1$.

Example 1 might suggest that lifting can always be performed when seeking to derive a linear valid inequality. Example 2 shows that it is not so.

Example 2. Consider the set $S=\left\{\left(x_{1}, x_{2}\right) \in[0,1]^{2} \mid-x_{1}^{2}-\left(x_{2}-0.5\right)^{2} \geq-0.5^{2}\right\}$.

The inequality $-x_{1} \geq 0$ is valid for the set $\left.S\right|_{x_{2}=0}$ obtained from $S$ by fixing $x_{2}=0$. By setting up an optimization problem similar to (2), it is easy to verify that there is no $\alpha \in \mathbb{R}$ for which $-x_{1}+\alpha x_{2} \geq 0$ is valid for $S$.

In Example 2, (i) set $S$ is convex, (ii) we are trying to lift a linear inequality, and (iii) $x_{2}$ is fixed to a bound. Even then, it is not possible to lift the seed inequality when we insist that lifting should be accomplished using an affine function of the fixed variable; see Example 4 in Section 3 for further discussion. 


\subsection{Sufficient conditions under which seed inequalities can be lifted}

In Theorem 1, we identify a large class of single row QCQPs where lifting can be accomplished using affine functions of the fixed variables.

Definition 1. A set $Q$ is a bipartite bilinear set1 if it is of the form

$$
S=\left\{(x, y) \in[0,1]^{m} \times[0,1]^{n} \mid x^{\top} Q y+a^{\top} x+b^{\top} y \geq c\right\}
$$

where $Q \in \mathbb{R}^{m \times n}, a \in \mathbb{R}^{m}, b \in \mathbb{R}^{n}$, and $c \in \mathbb{R}$.

Theorem 1. Let $S$ be a bipartite bilinear set. Given $C \times D \subset[m] \times[n]$ and $\tilde{x}_{i}, \tilde{y}_{j} \in\{0,1\}$ for $i \in[m] \backslash C$, $j \in[n] \backslash D$, assume that inequality $h\left(x_{C}, y_{D}\right) \geq r$ is valid for $\left\{(x, y) \in S \mid x_{[m] \backslash C}=\tilde{x}_{[m] \backslash C}, y_{[n] \backslash D}=\tilde{y}_{[n] \backslash D} \neq \neq \emptyset\right.$, where $h$ is a concave function defined on $[0,1]^{|C|+|D|}$. Then, for any $k \in[m] \backslash C$, there exists a finite $f_{k} \in$ $(-\infty, \infty)$ for which $h\left(x_{C}, y_{D}\right)+f_{k} x_{k} \geq r+f_{k} \tilde{x}_{k}$ is valid for $\left\{(x, y) \in S \mid x_{([m] \backslash C) \backslash\{k\}}=\tilde{x}_{([m] \backslash C) \backslash\{k\}}, y_{[n] \backslash D}=\right.$ $\tilde{y}_{[n] \backslash D\}}$.

Remark 1. The result of Theorem 1 can be applied iteratively to all the fixed variables one at a time to obtain a valid inequality for $S$. Theorem 1 holds even when the bounds on variables are not $[0,1]$, since we can always rescale and translate variables.

The proof of Theorem 1 is presented in Section 4 and uses calculations similar to those presented in Example 1. In particular, using a characterization of extreme points of the bipartite bilinear set $S$ [23, the proof reduces to establishing the result for three-variable problems where one of the variables is fixed. For a threevariable problem, a number of cases have to be analyzed to verify that the optimal value of an optimization problem similar to (2) is finite. The proof can be turned into an algorithm to compute the lifting coefficients, although not necessarily an efficient or practical one.

Theorem 1 assumes that, when variables $x$ and $y$ are fixed, they are fixed at their bounds (either 0 or 1.$)$ When this assumption is not imposed, we show next through an example that lifting may not be possible.

Example 3. Consider the bipartite bilinear set $S=\left\{(x, y, \hat{x}) \in[0,1]^{3} \mid(x-1 / 4)(y-1 / 2) \geq \hat{x} / 4+1 / 8\right\}$. First, we argue that the seed inequality $x \geq 3 / 4$ is valid for the restriction of $S$ where $\hat{x}=1 / 2$. This is clear as $|y-1 / 2| \leq 1 / 2$ when $y \in[0,1]$ and $|x-1 / 4|<1 / 2$ when $x<3 / 4$. Next, we claim that there is no $\alpha \in \mathbb{R}$ such that $x+\alpha(\hat{x}-1 / 2) \geq 3 / 4$ is valid for $S$. Assume by contradiction that $x+\alpha(\hat{x}-1 / 2) \geq 3 / 4$ is valid for $S$ for some $\alpha \in \mathbb{R}$. Since $(x, y, \hat{x})=(0,0,0) \in S$, we must have $-\alpha / 2 \geq 3 / 4$. Since $(x, y, \hat{x})=(1,1,1) \in S$, we must have $1+\alpha / 2 \geq 3 / 4$. This is the desired contradiction as the former expression requires that $\alpha \leq-3 / 2$ while the later requires that $\alpha \geq-1 / 2$.

\subsection{A framework for sequence-independent lifting}

Given a set of variables fixed at their bounds and a seed inequality for the corresponding restriction, a valid inequality for the original problem can be obtained by lifting each fixed variable one at the time. This computationally demanding process requires the solution of a non-convex nonlinear optimization problem, similar to (2), to lift each variable. It results in a lifted inequality whose form depends on the order in which variables are lifted. Next, we study situations where the lifting inequality obtained does not depend on the order in which variables are lifted. In particular, we develop a subadditive theory for lifting in QCQPs that is inspired by that originally developed in MILP in [55]. We consider the special case of separable bipartite bilinear constraints.

Definition 2. A set $Q$ is a separable bipartite bilinear set if it is of the form

$$
Q:=\left\{(x, y) \in[0,1]^{n} \times[0,1]^{n} \mid \sum_{i=1}^{n} a_{i} x_{i} y_{i} \geq d\right\},
$$

for some $d$ and $a_{i} \in \mathbb{R}$ for $i \in[n]$, i.e., variables $x_{i}$ and $y_{i}$, for $i \in[n]$, appear in only one term.

\footnotetext{
${ }^{1}$ We use the term bipartite, perhaps redundantly, to highlight that variables can be divided into two groups, such that any degree two term comes from product of variables one each from these two groups [23].
} 
In the separable case, it is natural to lift each pair of variables $x_{i}$ and $y_{i}$ together. Next, we derive conditions that guarantee that the form of the lifted inequality obtained is independent of the order in which these pairs are lifted. This result is obtained, as is common in MILP, by deriving a subadditive upper bound on the lifting function of the seed inequality, from which all lifting coefficients can be derived.

Definition 3. Let $Q$ be a separable bipartite bilinear set. Assume that $\Lambda=\left\{I, J_{0}, J_{1}\right\}$ is a partition of $[n]$ (i.e., $I \cup J_{0} \cup J_{1}=[n]$ with $I \cap J_{0}=I \cap J_{1}=J_{0} \cap J_{1}=\emptyset$ ) and that $h\left(x_{I}, y_{I}\right) \geq r$, is a valid inequality for $\left\{(x, y) \in Q \mid x_{J_{0}}=y_{J_{0}}=0, x_{J_{1}}=y_{J_{1}}=1\right\}$. For $\delta \in \mathbb{R}$, we define the lifting function of the seed inequality as

$$
\phi(\delta):=\max \left\{r-h\left(x_{I}, y_{I}\right) \mid \sum_{i \in I} a_{i} x_{i} y_{i} \geq\left(d-\sum_{i \in J_{1}} a_{i}\right)-\delta,\left(x_{I}, y_{I}\right) \in[0,1]^{2|I|}\right\} .
$$

Structured approximations of lifting functions allow for simple lifting of inequalities as described next in Proposition 1 whose proof can be found in Section 5.

Proposition 1. Let $Q$ be a separable bipartite bilinear set and let $\Lambda=\left\{I, J_{0}, J_{1}\right\}$ be a partition of $[n]$. Let $\phi$ be the lifting function of seed inequality $h\left(x_{I}, y_{I}\right) \geq r$ for $\left\{(x, y) \in Q \mid x_{J_{0}}=y_{J_{0}}=0, x_{J_{1}}=y_{J_{1}}=1\right\}$ where $h$ is a concave function. Assume there exists $\psi: \mathbb{R} \mapsto \mathbb{R}$ and concave functions $\gamma_{i}: \mathbb{R}^{2} \mapsto \mathbb{R}$ for $i \in J_{0} \cup J_{1}$ such that

(i) $\psi(\delta) \geq \phi(\delta), \forall \delta \in \mathbb{R}$;

(ii) $\psi$ subadditive, (i.e., $\psi\left(\delta_{1}\right)+\psi\left(\delta_{2}\right) \geq \psi\left(\delta_{1}+\delta_{2}\right), \forall \delta_{1}, \delta_{2} \in \mathbb{R}$ ) with $\psi(0)=0$;

(iii) for $i \in J_{0}, \gamma_{i}(x, y) \geq \psi\left(a_{i} x y\right), \forall(x, y) \in[0,1]^{2}$,

(iv) for $i \in J_{1}, \gamma_{i}(x, y) \geq \psi\left(a_{i} x y-a_{i}\right), \forall(x, y) \in[0,1]^{2}$.

Then, the lifted inequality $h\left(x_{I}, y_{I}\right)+\sum_{i \in J_{0} \cup J_{1}} \gamma_{i}\left(x_{i}, y_{i}\right) \geq r$ is a valid convex inequality for $Q$.

The statement of Proposition 1 does not specify the type of functional forms $\gamma_{i}\left(x_{i}, y_{i}\right)$ to use in ensuring that conditions (iii) and (iv) are satisfied. It is however clear from the definition that choosing $\gamma_{i}\left(x_{i}, y_{i}\right)$ to be the concave envelope of $\psi\left(a_{i} x_{i} y_{i}\right)$ over $[0,1]^{2}$ when $i \in J_{0}$, and the concave envelope of $\psi\left(a_{i} x_{i} y_{i}-a_{i}\right)$ over $[0,1]^{2}$ when $i \in J_{1}$ is the preferred choice for $\gamma_{i}$.

Remark 2. While we state the result of Proposition 1 for a set $Q$ defined by a single separable bipartite bilinear constraint, a similar result would also hold for sets defined by multiple separable bipartite bilinear constraints.

\subsection{A seed inequality from a minimal covering set}

To generate lifted inequalities for separable bipartite bilinear sets, we focus next on a family of restrictions we refer to as minimal covering sets. For such minimal covering sets, we introduce a provably strong convex, second-order cone representable valid inequality. We use this inequality as the seed in our lifting procedures.

Definition 4. Let $k \in \mathbb{Z}_{+}$be a positive integer. We say that $a_{i} \in \mathbb{R}$ for $i \in[k]$ form a minimal cover of $d \in \mathbb{R}$, if (i) $a_{i}>0$ for all $i \in[k], d>0$, (ii) $\sum_{i=1}^{k} a_{i}>d$, (iii) $\sum_{i \in K} a_{i} \leq d, \forall K \subsetneq[k]$. For a separable bipartite bilinear set $Q$, we say that a partition $\Lambda=\left\{I, J_{0}, J_{1}\right\}$ of $[n]$, where $I \neq \emptyset$, is a minimal cover yielding partition if: $a_{i}$ for $i \in I$ form a minimal cover of $d^{\Lambda}:=d-\sum_{i \in J_{1}} a_{i}$. For a minimal cover yielding partition, we let $J_{0}^{+}:=\left\{i \in J_{0} \mid a_{i}>0\right\}, J_{0}^{-}:=\left\{i \in J_{0} \mid a_{i}<0\right\}$; we define $J_{1}^{+}$and $J_{1}^{-}$similarly.

Remark 3. When $k \geq 2$, conditions (ii) and (iii) in the definition of minimal cover imply condition (i) For example, if $a_{i} \leq 0$ for some $i \in[k]$, then (ii) implies $\sum_{j \in[k] \backslash\{i\}} a_{j}>d$, contradicting (iii). Now (iii) together with $a_{i}>0$ for $i \in[k]$ implies $d>0$.

Notation 1. Assuming that $a_{i}$ for $i \in[n]$ form a minimal cover of $d$, we use (i) $\Delta:=\sum_{i=1}^{n} a_{i}-d$, (ii) $d_{i}:=d-\sum_{j \in[n] \backslash\{i\}} a_{j}$, (iii) $I^{>}:=\left\{i \in[n] \mid a_{i}>\Delta\right\}$, (iv) when $I^{>} \neq \emptyset$, $i_{0}$ to be any index in $I^{>}$such that $a_{i_{0}}=\min \left\{a_{i} \mid i \in I^{>}\right\}$. 
For a minimal cover, conditions (ii) and (iii) in Definition 4 imply that $\Delta>0$ and $a_{i} \geq \Delta$ for all $i \in[n]$, respectively. Simple computations show that $d_{i}=a_{i}-\Delta$.

Our overall plan is the following. We will fix $x_{i}=y_{i}=0$ for $i \in J_{0}$ and $x_{i} y_{i}=1$ for $i \in J_{1}$. Then, we will find a valid seed inequality for the set where the coefficients form a minimal cover. Finally, we will lift this seed inequality. One key reason to generate cuts from a seed inequality corresponding to a minimal cover is the following result.

Theorem 2. For a nonempty separable bilinear set $Q$, either there exists at least one minimal cover yielding partition or $\operatorname{conv}(Q)$ is polyhedral.

Loosely speaking, the proof of Theorem 2, which is given in Section 6, is based on showing that if there is no minimal cover yielding partition, then $Q$ is "almost" a packing-type set, i.e., a set of the form $\{(x, y) \in$ $\left.[0,1]^{2 n} \mid \sum_{i=1}^{n} a_{i} x_{i} y_{i} \leq d\right\}$ where $a_{i}$ s are non-negative. For packing sets $Q,\left[51\right.$, shows that $\operatorname{conv}(Q)=\operatorname{proj}_{x, y}(G)$ where

$$
G=\left\{(x, y, w) \in[0,1]^{3 n} \mid \sum_{i=1}^{n} a_{i} w_{i} \leq d, x_{i}+y_{i}-1 \leq w_{i}, \forall i \in[n]\right\} .
$$

We say "almost", since there are non-packing sets such as $S:=\left\{(x, y) \in[0,1]^{4} \mid x_{1} y_{1}-100 x_{2} y_{2} \geq-98\right\}$, where there is no partition that yields a minimal cover. Such sets are "overwhelmingly" like a packing set; in the case of the example, it is a perturbation of the packing set $\left\{\left(x_{2}, y_{2}\right) \in[0,1]^{2} \mid 100 x_{2} y_{2} \leq 98\right\}$. For such sets it is not difficult to show that $\operatorname{conv}(S)$ is polyhedral.

Since the main focus of this paper is the study of lifted convex (nonlinear) inequalities and since in the packing case the convex hull is trivially obtained using McCormick inequalities [39, the remainder of the paper will concentrate on the case where there exists a minimal cover yielding partition.

Associated with a minimal cover is a convex valid inequality that we present next.

Theorem 3. Consider the separable bipartite bilinear minimal covering set as presented in Definition 2 where $a_{i}, i \in[n]$ form a minimal cover of $d$. Then, the bilinear cover inequality is valid for $Q$ :

$$
\sum_{i=1}^{n} \frac{\sqrt{a_{i}}}{\sqrt{a_{i}}-\sqrt{d_{i}}}\left(\sqrt{x_{i} y_{i}}-1\right) \geq-1 .
$$

Our proof of Theorem 3 , which is presented in Section 7 , uses techniques from disjunctive programming 8 and an "approximate version" of Fourier-Motzkin projection. In particular, using the minimal covering property of the coefficients and a characterization of the extreme points of bipartite bilinear sets [23], we obtain $n$ secondorder cone representable sets whose union contains all the extreme points separable bipartite bilinear set. Next we write an extended formulation [8, 15] of the convex hull of the union of these sets. Finally, we use the Fourier-Motzkin procedure to project out the auxiliary variables of the extended formulation one at a time. This procedure works to project out most of the variables. The last step however requires a relaxation to be constructed so that projection can be carried in closed-form. Finally we obtain an inequality that is in fact stronger than (3).

Inequality (3) can be viewed as a strengthening of an inequality presented in [53] for the set $Q^{\text {relax }}$ obtained from $Q$ by relaxing upper bounds on variables, i.e., $Q^{\text {relax }}:=\left\{(x, y) \in \mathbb{R}_{+}^{2 n} \mid \sum_{i=1}^{n} a_{i} x_{i} y_{i} \geq d\right\}$, where $a_{i}>0$ for $i \in[n]$ and $d>0$. The convex hull of $Q^{\text {relax }}$ is shown in [53] to be described by nonnegativity constraints together with

$$
\sum_{i=1}^{n} \frac{\sqrt{a_{i}}}{\sqrt{d}} \sqrt{x_{i} y_{i}} \geq 1
$$

The ensuing proposition, whose proof we skip due to lack of space shows that (3) improves on (4). It essentially proceeds by comparing the coefficients of variable pairs $\sqrt{x_{i} y_{i}}$ inside of the inequalities. Moreover, if $n \geq 2$ and there exists $i \in[n]$ such that $d_{i}>0$, then (3) strictly dominates (4).

Proposition 2. Inequality (4) is dominated by (3) over the $0-1$ box, i.e.,

$$
\left\{(x, y) \in[0,1]^{2 n} \mid(4)\right\} \supseteq\left\{(x, y) \in[0,1]^{2 n} \mid(3)\right\} .
$$


Even though Proposition 2 hints at the strength of the bilinear cover inequality, it can be easily verified that (3) does not produce the convex hull of $Q$. However there are a number of reasons to use this inequality as a seed for lifting. The first reason is that, not only is inequality (3) second-order cone representable, we only need to introduce one extra variable representing $\sqrt{x_{i} y_{i}}$ for each $i \in[n]$, to write it as a second-order cone representable set. Apart from the convenience of using this inequality within modern conic solvers, the main reason for considering it as a seed inequality is its strength. In particular, we prove next that (3) provides a constant factor approximation of the convex hull of the original set.

Theorem 4. Let $Q$ be a bipartite bilinear minimal covering set Let $R:=\left\{(x, y) \in \mathbb{R}_{+}^{2 n} \mid(3)\right\}$. Then $(4 \cdot R) \cap$ $[0,1]^{2 n} \subseteq \operatorname{conv}(Q) \subseteq R \cap[0,1]^{2 n}$.

Since $R$ is a set of the covering type (that is, its recession cone is the non-negative orthant), we have that $4 \cdot R \subseteq R$. The proof of Theorem 4, which is given in Section 8, is based on optimizing linear functions with non-negative coefficients on $R$ and $Q$ and proving a bound of 4 on the ratio of their optimal objective function values.

\subsection{Lifting the bilinear cover inequality}

We now follow the framework of Proposition 1 to perform sequence-independent lifting of the bilinear cover inequality. The first step is to study the lifting function.

Theorem 5. Let $\phi$ be the lifting function for valid inequality (3). Define

$$
\psi(\delta):=\left\{\begin{array}{lrl}
l_{+}(\delta+\Delta)-1 & \delta & \leq-\Delta \\
l_{-} \delta & -\Delta \leq \delta & \leq 0 \\
l_{+} \delta & 0 \leq \delta,
\end{array}\right.
$$

where $l_{-}=\frac{1}{\Delta}$ and where $l_{+}=\frac{\sqrt{a_{i_{0}}}+\sqrt{d_{i_{0}}}}{\Delta \sqrt{d_{i_{0}}}}$ if $a_{i_{0}}$ exists and $l_{+}=\frac{1}{\Delta}$ otherwise. Then

(i) $l_{+} \geq l_{-}>0$,

(ii) $\psi(\delta)$ is subadditive over $\mathbb{R}$ with $\psi(0)=0$, and

(iii) $\phi(\delta) \leq \psi(\delta)$ for $\delta \in \mathbb{R}$.

Although computing the lifting function for an arbitrary valid inequality, in general, appears to be a difficult task, the bilinear cover inequality (3) has sufficient structure that we can derive a strong subadditive upper bound in Theorem 5 The key to proving Theorem [5, as we show in Section 9, is to first obtain the lifting function exactly in a region around the origin, and to argue that the linear upper bound of the lifting function for this region upper bounds the lifting function globally. Figure 1 presents examples of the lifting function $\phi$, and the upper bound $\psi$ we derived in Theorem 5 for the cases when $a_{i_{0}}$ exists and for the case when it does not.

We observe in Figure 1 that the lifting function is not subadditive since it is convex in a neighborhood of the origin. Therefore, building a subadditive approximation is required to achieve sequence-independent lifting.

Building on the subadditive upper bound obtained in Theorem 5, we are now able to lift the bilinear cover inequality in a sequence-independent manner.

Theorem 6. Consider the separable bipartite bilinear set presented in Definition 2. Let $\Lambda=\left\{I, J_{0}, J_{1}\right\}$ be a minimal cover yielding partition and let $\Delta, a_{i_{0}}, d_{i}, l_{+}, l_{-}$be defined as in Theorems 3 and 5 (We clarify that they are calculated using $d^{\Lambda}$ instead of $d$ ). Let $J_{0}^{+}, J_{0}^{-}, J_{1}^{+}$, and, $J_{1}^{-}$be as in Definition 4. Then inequality

$$
\sum_{i \in I} \frac{\sqrt{a_{i}}}{\sqrt{a_{i}}-\sqrt{d_{i}}}\left(\sqrt{x_{i} y_{i}}-1\right)+\sum_{i \notin I} \gamma_{i}\left(x_{i}, y_{i}\right) \geq-1,
$$

is valid for $Q$ where $\gamma_{i}: \mathbb{R}^{2} \rightarrow \mathbb{R}$ for $i \in[n] \backslash I$ are the concave functions:

$$
\text { (i) } \gamma_{i}(x, y)=l_{+} a_{i} \min \{x, y\} \text { for } i \in J_{0}^{+} \text {; }
$$




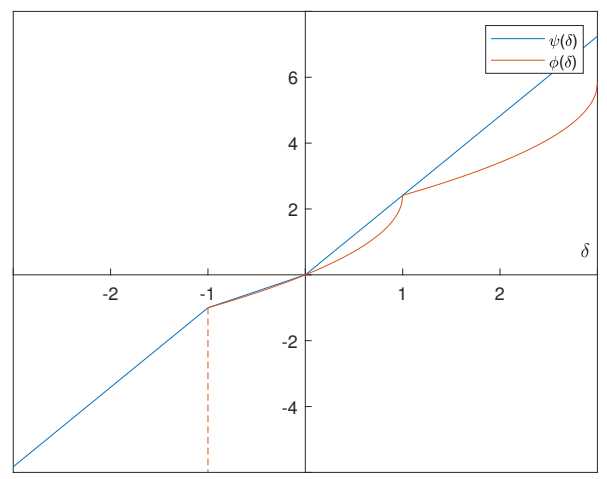

(a) $a_{i}=2, \Delta=1$

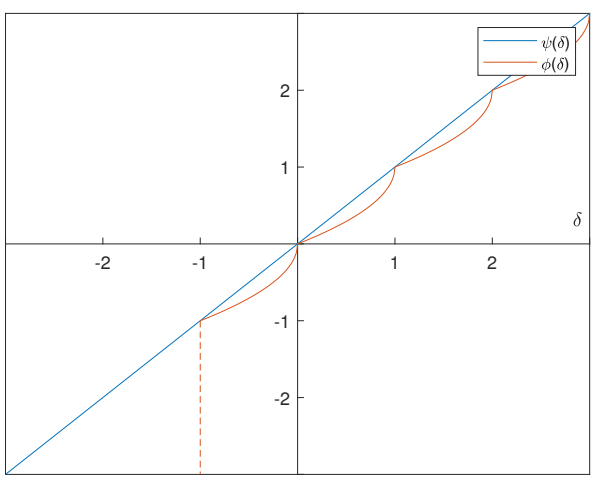

(b) $a_{i}=1, \Delta=1$

Figure 1: Lifting function $\phi(\delta)$ in red and subadditive upper bound $\psi(\delta)$ in blue

(ii) $\gamma_{i}(x, y)=-l_{+} a_{i} \min \{2-x-y, 1\}$ for $i \in J_{1}^{-}$;

(iii) $\gamma_{i}(x, y)=\min \left\{l_{-} a_{i}(x+y-1), l_{+} a_{i}(x+y-1)+l_{+} \Delta-1,0\right\}$ for $i \in J_{0}^{-}$;

(iv) $\gamma_{i}(x, y)=\min \left\{\tilde{g}_{i}(x, y), \tilde{h}_{i}(x, y), g_{i}(x, y), h_{i}(x, y)\right\}$, for $i \in J_{1}^{+}$with $a_{i} \geq a_{i_{0}}$ when $I^{>} \neq \emptyset$, and $\gamma_{i}(x, y)=$ $\min \left\{\tilde{g}_{i}(x, y), \tilde{h}_{i}(x, y)\right\}$ in all other cases where $i \in J_{1}^{+}$, with

$$
\begin{aligned}
\tilde{g}_{i}(x, y) & =l_{+} a_{i}(\min \{x, y\}-1)+l_{+} \Delta-1 \\
\tilde{h}_{i}(x, y) & =l_{-} a_{i}(\min \{x, y\}-1) \\
g_{i}(x, y) & =\sqrt{a_{i}-\Delta} \sqrt{a_{i}} l_{+} \sqrt{x y}-l_{+}\left(a_{i}-\Delta\right)-1 \\
h_{i}(x, y) & =\frac{\sqrt{a_{i}}}{\sqrt{a_{i}}-\sqrt{d_{i}}}(\sqrt{x y}-1) .
\end{aligned}
$$

We refer to inequality (6) as lifted bilinear cover inequality. This inequality is second-order cone representable. The proof of Theorem 6 can be found in Section 10.

\section{Future directions}

The results presented in this paper open up new avenues for generating cutting-planes for QCQPs. They also raise new theoretical and computational questions that can be investigated. To illustrate this assertion, we revisit Example 2 next.

Example 4. Consider $S:=\left\{\left(x_{1}, x_{2}\right) \in[0,1]^{2} \mid-x_{1}^{2}-\left(x_{2}-0.5\right)^{2} \geq-0.5^{2}\right\}$ with the same fixing as in Example 2 . i.e., $x_{2}=0$. For the associated restriction $\left.S\right|_{x_{2}=0}$, consider the seed inequality $-x_{1} \geq 0$.

In contrast to our earlier discussion, consider now the problem of lifting this seed inequality into an inequality of the form $-x_{1}+\alpha \sqrt{x_{2}} \geq 0$. Finding the values of $\alpha$ that generate a valid inequality is equivalent to solving the problem

$$
\alpha^{*}:=\sup \left\{\frac{x_{1}}{\sqrt{x_{2}}} \mid-x_{1}^{2}-\left(x_{2}-0.5\right)^{2} \geq-0.5^{2}, x_{1} \in[0,1], x_{2} \in(0,1]\right\} \text {. }
$$

Using constraint $-x_{1}^{2}-\left(x_{2}-0.5\right)^{2} \geq-0.5^{2}$ we can bound the objective function as: $\frac{x_{1}}{\sqrt{x_{2}}} \leq \frac{\sqrt{0.5^{2}-\left(x_{2}-0.5\right)^{2}}}{\sqrt{x_{2}}}=$ $\frac{\sqrt{\left(1-x_{2}\right)\left(x_{2}\right)}}{\sqrt{x_{2}}}=\sqrt{1-x_{2}}$. It follows that selecting $\alpha \geq \alpha^{*}=1$ yields a valid inequality for $S$. Note first that $\alpha<0$ leads to an invalid inequality since $\left(x_{1}, x_{2}\right)=(0,0.5)$ is a feasible point. Moreover, any $\alpha \in[0,1)$ yields 
an invalid inequality, since the point $\left(x_{1}, x_{2}\right)$ where $x_{1}=\sqrt{x_{2}\left(1-x_{2}\right)}$ and $x_{2}=1-((1+\alpha) / 2)^{2}$ is feasible. Therefore, the inequality $-x_{1}+\sqrt{x_{2}} \geq 0$ is the strongest such lifted inequality.

The above example raises the question of obtaining a complete characterization of when one can accomplish lifting, i.e., of generalizing Theorem 1 to situations where the functional form of the lifted variable is not necessarily linear. It would also be valuable to develop a theory to accomplish sequence-independent lifting in the more general case of bipartite bilinear programs, instead of just the separable case. On the computational side, one key question is to understand the complexity of separating the lifted bilinear cover inequality presented in Theorem 6 and to design efficient computational schemes to perform separation. Finally, extensive numerical experiments should be conducted to understand the practical strength of these inequalities and to determine how useful they can be in the solution of QCQPs. Given the strength of the seed inequality, we are hopeful that these lifted inequalities could yield nontrivial dual bound improvements.

\section{Proof of Theorem 1}

Theorem 1. Without loss of generality, we assume that we lift a component of the variable $x$, say $x_{k}$ with $k \in[m] \backslash C$. In addition, we assume $\tilde{x}_{k}=0$; if not we may perform the operation $x_{k} \leftarrow 1-x_{k}$ and $f_{k} \leftarrow-f_{k}$.

In order to find a lifting coefficient, We examine the following optimization problem

$$
\begin{aligned}
u_{k}\left(x_{k}\right):=\frac{1}{x_{k}} \quad \max & r-h\left(x_{C}, y_{D}\right) \\
\text { s.t. } \quad & x^{\boldsymbol{\top}} Q y+a^{\boldsymbol{\top}} x+b^{\boldsymbol{\top}} y \geq c, \\
& x_{C}, y_{D} \in[0,1], x_{[m] \backslash C \backslash\{k\}}=\tilde{x}_{[m] \backslash C \backslash\{k\}}, y_{[n] \backslash D}=\tilde{y}_{[n] \backslash D} .
\end{aligned}
$$

Now note that $u_{k}^{*}=\sup _{x_{k} \in(0,1]} u_{k}\left(x_{k}\right)$, assuming it exists, is a valid the coefficient for lifting, i.e., $h\left(x_{C}, y_{D}\right)+$ $u_{k}^{*} x_{k} \geq r$ is a valid lifted inequality. Any coefficient larger than $u_{k}^{*}$ is also valid for lifting.

From the concavity of $h$ (i.e., convexity of $r-h$ ), for any specific $x_{k}$ the optimal solution must be an extreme point. According to [23], all extreme points satisfy the following property: except one pair of $\left(x_{i}, y_{j}\right)$, all other $x_{i^{\prime}}, y_{j^{\prime}}$ pairs will be equal to either 0 or 1 . Thus, for any pair of partitions $\{i\} \cup I_{0} \cup I_{1}=C$ (denoted by $I$ ) and $\{j\} \cup J_{0} \cup J_{1}=D($ denoted by $J)$, define

$$
\begin{aligned}
u_{I, J}\left(x_{k}\right):=\frac{1}{x_{k}} \quad \max & r-h\left(x_{C}, y_{D}\right) \\
\text { s.t. } \quad & x^{\top} Q y+a^{\boldsymbol{\top}} x+b^{\top} y \geq c, \\
& x_{i}, y_{j} \in[0,1], x_{I_{0}}=0, x_{I_{1}}=1, y_{J_{0}}=0, y_{J_{1}}=1, \\
& x_{[m] \backslash C \backslash\{k\}}=\tilde{x}_{[m] \backslash C \backslash\{k\}}, y_{[n] \backslash D}=\tilde{y}_{[n] \backslash D} .
\end{aligned}
$$

We clearly have $u_{k}\left(x_{k}\right)=\max _{I, J} u_{I, J}\left(x_{k}\right)$. In addition, observe that $u_{k}^{*}=\max _{I, J} u_{I, J}^{*}$ where $u_{I, J}^{*}=\sup _{x_{k} \in(0,1]} u_{I, J}\left(x_{k}\right)$. Therefore in order to prove that $u_{k}^{*}<\infty$, it is sufficient to show that for any partition $I, J, u_{I, J}^{*}<\infty$. Therefore, we now focus on one instance of such partitions.

We define $\tilde{x} \in \mathbb{R}^{m}$ and $\tilde{y} \in \mathbb{R}^{n}$ as: $\left(\tilde{x}_{I, J}\right)_{I_{0} \cup\{i\}}=0,\left(\tilde{x}_{I, J}\right)_{I_{1}}=1,\left(\tilde{y}_{I, J}\right)_{J_{0} \cup\{j\}}=0,\left(\tilde{y}_{I, J}\right)_{J_{1}}=1$. In addition, define $r_{I, J}:=r-p_{I_{1}}^{\top} \mathbf{1}_{I_{1}}-q_{J_{1}}^{\top} \mathbf{1}_{J_{1}}, c_{I, J}:=c-a^{\top} \tilde{x}_{I, J}-b^{\top} \tilde{y}_{I, J}-\tilde{x}_{I, J}^{\top} Q \tilde{y}_{I, J}, a_{I, J}:=a_{i}+Q_{i, *} \tilde{y}_{I, J}$, $b_{I, J}:=b_{j}+\tilde{x}_{I, J}^{\top} Q_{*, j}$, and $a_{I, J, k}:=a_{k}+Q_{k, *} \tilde{y}_{I, J}$ so that we have equivalently

$$
\begin{array}{rll}
u_{I, J}\left(x_{k}\right)=\frac{1}{x_{k}} \quad \max & r_{I, J}-h_{I, J}\left(x_{i}, y_{j}\right) \\
\text { s.t. } \quad & q_{i j} x_{i} y_{j}+a_{I, J} x_{i}+b_{I, J} y_{j}+a_{I, J, k} x_{k}+q_{k j} x_{k} y_{j} \geq c_{I, J}, \\
& \left(x_{i}, y_{j}\right) \in[0,1]^{2},
\end{array}
$$

where $h_{I, J}$ is $h$ after the appropriate restriction. Note that $h_{I, J}$ is concave. As we are focusing on the pair of partitions $I, J$, for simplicity we rewrite the problem as

$$
\begin{array}{rll}
u(\hat{x}):=\frac{1}{\hat{x}} \quad \max _{x, y} & r-h(x, y) \\
& \text { s.t. } \quad q x y+a x+b y+\hat{a} \hat{x}+\hat{q} \hat{x} y \geq c,(x, y) \in[0,1]^{2},
\end{array}
$$


and $u^{*}:=\sup _{\hat{x} \in(0,1]} u(\hat{x})$. It remains to prove $u^{*}<\infty$.

For any $\epsilon \in(0,1]$ and $\hat{x} \in[\epsilon, 1]$, we have

$$
\begin{aligned}
u(\hat{x}) & =\max _{x, y} \quad\left\{\frac{1}{\hat{x}}(r-h(x, y)) \mid q x y+a x+b y+\hat{a} \hat{x}+\hat{q} \hat{x} y \geq c,(x, y) \in[0,1]^{2}\right\}, \\
& \leq \max _{x, y} \quad\left\{\frac{1}{\hat{x}}(r-h(x, y)) \mid(x, y) \in[0,1]^{2}\right\} \\
& \leq \max _{x, y}\left\{\max \left\{\frac{1}{\epsilon}(r-h(x, y)),(r-h(x, y))\right\} \mid(x, y) \in[0,1]^{2}\right\} \\
& :=w<\infty .
\end{aligned}
$$

It is clear that $u(\hat{x}) \leq w<\infty$ for any $\hat{x} \in[\epsilon, 1]$. Therefore, to show that $u^{*}<\infty$, it is sufficient to show that $\lim \sup _{\hat{x} \downarrow 0} u(\hat{x})<\infty$. We define

$$
\begin{aligned}
& v(\hat{x})=\max r-h(x, y) \\
& \text { s.t. } x \geq 0, \\
& x \leq 1, \\
& y \geq 0, \\
& y \leq 1, \\
& \\
& q x y+a x+b y+\hat{a} \hat{x}+\hat{q} \hat{x} y \geq c .
\end{aligned}
$$

Denote the feasible region of (7) as $S(\hat{x})$. Since $v(0) \leq 0$ (because the seed inequality is assumed to be valid for

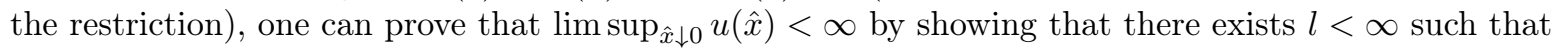

$$
v(\hat{x})-v(0) \leq l \hat{x}+o(\hat{x}) \text { for } \hat{x} \downarrow 02
$$

We denote the feasible region of the above problem as $S(\hat{x})$.

For $i \in\{1, \ldots, 5\}$, we define $v_{i}(\hat{x})$ to be the optimal value of $(7)$ where constraint $(7 i)$ is at to equality. We use $S_{i}(\hat{x})$ to denote the corresponding feasible region. For example,

$$
\begin{aligned}
v_{a}(\hat{x}) & =\max \left\{r-h(x, y) \mid(x, y) \in S_{1}(\hat{x})\right\} \\
& =\max \{r-h(x, y) \mid x=0,(x, y) \in S(\hat{x})\} \\
& =\max \{r-h(0, y) \mid b y+\hat{a} \hat{x}+\hat{q} \hat{x} y \geq c, y \in[0,1]\} .
\end{aligned}
$$

Note that $v(\hat{x})=\max _{i \in\{1, \ldots, 5\}}\left\{v_{i}(\hat{x})\right\}$, since the objective function in computing $v(\hat{x})$ is maximizing a convex function, implying that there exists an optimal solution where at least one of the constraints (7.1)-(7.5) is active.

Thus, to prove that $\lim \sup _{\hat{x} \downarrow 0} u(\hat{x})<\infty$ it suffices to show that there exists $l<\infty$ such that

$$
v_{i}(\hat{x})-v(0) \leq l \hat{x}+o(\hat{x}) \text { for } \hat{x} \downarrow 0 \text { for all } i \in\{1, \ldots, 5\} .
$$

The case of $v_{1}, v_{2}, v_{3}, v_{4}$ : We present a proof of (8) for the case of $v_{1}$. The proof is similar for the cases of $v_{2}, v_{3}$, and $v_{4}$.

First it is straightforward to verify that there exists a sufficiently small $\hat{x}_{0}>0$ such that for any $0<\hat{x}<\hat{x}_{0}$, we have one of the following two cases: (i) $S_{1}(\hat{x}) \backslash S_{1}(0)=\emptyset$ (including the case $\left.S_{1}(\hat{x})=\emptyset\right)$ and (ii) $S_{1}(\hat{x}) \backslash S_{1}(0) \neq$ $\emptyset$, as, for sufficiently small $\hat{x}_{0}$, we may assume that it is impossible that $S_{1}(\hat{x}) \neq \emptyset=S_{1}(0)$.

(i) We have $v_{1}(\hat{x}) \leq v_{1}(0) \leq v(0) \leq 0$ (this holds even in the case when $S_{1}(\hat{x})=\emptyset$ or $S_{1}(\hat{x})=S_{1}(0)=\emptyset$ ), i.e., $v_{1}(\hat{x})-v(0) \leq 0 \cdot \hat{x}$.

(ii) We consider two sub-cases:

(a) if $b=0$, the feasibility of $\hat{x}=0$ yields $c \leq 0$. Thus, we have $S_{1}(0)=[0,1] \supseteq S_{1}(\hat{x})$ for $\hat{x} \in\left(0, \hat{x}_{0}\right)$ (actually in case (i) .

\footnotetext{
${ }^{2}$ This is equivalent to saying $\lim \sup _{\hat{x} \downarrow 0} \frac{v(\hat{x})-v(0)}{\hat{x}} \leq l$
} 
(b) if $b \neq 0$, assume first that $b<0$. Then $S_{1}(\hat{x})=\{y \in[0,1]: y \leq(c-\hat{a} \hat{x}) /(b+\hat{q} \hat{x})\}$. We also denote $\Delta(\hat{x}):=(c-\hat{a} \hat{x}) /(b+\hat{q} \hat{x})-c / b$ and since $b<0$, we have that $|\mathrm{d} \Delta(\hat{x}) / \mathrm{d} \hat{x}|<\infty$ for $\hat{x}=0$.

Since $S_{1}(\hat{x}) \backslash S_{1}(0) \neq \emptyset$, we have $0 \leq c / b<1$ as well as $\Delta(\hat{x}) \geq 0$.

Utilizing the fact that $\Delta(\hat{x}) \in[0,1-c / b]$ (the upper bound from the fact that $\hat{x}$ is assumed to be sufficiently small) and the concavity of $h$, we obtain

$$
h\left(0, \frac{c}{b}+\Delta(\hat{x})\right) \geq \frac{\Delta(\hat{x})}{1-c / b} h(0,1)+\left(1-\frac{\Delta(\hat{x})}{1-c / b}\right) h\left(0, \frac{c}{b}\right) .
$$

We now have

$$
\begin{aligned}
v_{1}(\hat{x})-v(0) \leq & v_{1}(\hat{x})-v_{1}(0) \\
\leq & \max \{(r-h(0,0))-(r-h(0,0)), \\
& (r-h(0, \Delta(\hat{x})+c / b)-(r-h(0, c / b))\} \\
\leq & \max \left\{0, \frac{h(0, c / b)-h(0,1)}{1-c / b} \Delta(\hat{x})\right\} \\
= & \max \left\{0,\left.\frac{h(0, c / b)-h(0,1)}{1-c / b} \frac{\mathrm{d} \Delta(\hat{x})}{\mathrm{d} \hat{x}}\right|_{\hat{x}=0} \hat{x}+o(\hat{x})\right\},
\end{aligned}
$$

where the second inequality comes from the fact that $v_{1}(\hat{x})=\max \{r-h(0,0), r-h(0, \Delta+c / b)\}$ and $v_{1}(0)=\max \{r-h(0,0), r-h(0, c / b)\}$, and the third inequality follows from $(9)$, and the last equality follows from Taylor's series expansion of $\frac{h(0, c / b)-h(0,1)}{1-c / b} \Delta(\hat{x})$ around $\hat{x}=0$.

Thus, there exists $l_{1}<\infty$ such that $v_{1}(\hat{x})-v(0) \leq l_{1} \hat{x}+o(\hat{x})$ for $\hat{x} \downarrow 0$. A similar argument holds for the case of $b>0$.

The case of $v_{5}:$ If $q=0$, then it is easy to see that there always exists an optimal solution to the optimization problem corresponding to computing $v(\hat{x})$ such that one of (7.1)-(7.4) is active. Therefore if $q=0$, it is sufficient to verify (8) for $v_{1}, v_{2}, v_{3}$, and $v_{4}$ as $v_{5}(\hat{x}) \leq \max \left\{v_{1}(\hat{x}), v_{2}(\hat{x}), v_{3}(\hat{x}), v_{4}(\hat{x})\right\}$.

Therefore, we consider the case of $v_{5}$ for $q \neq 0$. Without loss of generality assume that $q>0$ or perform the transformation $x \leftarrow 1-x$. We in addition assume that $q=1$ or we can scale all parameters by $1 / q$. The problem can now be rewritten as

$$
\begin{aligned}
v_{5}(\hat{x}):=\max & r-h(x, y) \\
& \text { s.t. } \quad(x+b+\hat{q} \hat{x})(y+a)=c+a b+(\hat{q} a-\hat{a}) \hat{x}, \quad(x, y) \in[0,1]^{2} .
\end{aligned}
$$

We denote its feasible region by $S_{5}(\hat{x})$.

The feasible region is the boundary of a hyperbola intersected with the $[0,1]^{2}$ box. If both the connected components of the hyperbola intersect the $[0,1]^{2}$ box, or $c+a b+(\hat{q} a-\hat{a}) \hat{x} \leq 0$, then it is easy to see that there exists an optimal solution of the optimization problem corresponding to computation of $v(\hat{x})$ where at least one of (7.1)-(7.4) is active, i.e., $v_{5}(\hat{x}) \leq \max \left\{v_{1}(\hat{x}), v_{2}(\hat{x}), v_{3}(\hat{x}), v_{4}(\hat{x})\right\}$. So we can disregard this case as well and assume that only one of the connected components of the hyperbola is feasible, as well as $c+a b+(\hat{q} a-\hat{a}) \hat{x} \geq 0$.

Note again that if $S(0)=\emptyset$, then there exists $\hat{x}_{0}>0$ such that $S(\hat{x})=\emptyset$ for all $0 \leq \hat{x}<\hat{x}_{0}$ and thus $v_{5}(\hat{x}) \leq v(\hat{x}) \leq 0$. Therefore we may assume that $S(0) \neq \emptyset$. Let $h^{M}:=\max \left\{\max _{(x, y) \in[0,1]^{2}} h(x, y), 0\right\}$ and $h^{m}=\min \left\{\min _{(x, y) \in[0,1]^{2}} h(x, y), 0\right\}$.

(i) We first consider the case when $c+a b=0$. Feasibility of $S(0)$ requires $-b \in[0,1]$ or $-a \in[0,1]$. In addition, as only one part of the hyperbola is feasible for $S(\hat{x})$ for $\hat{x}>0$ (and sufficiently small), we obtain that either $-a \notin(0,1)$ or $-b \notin(0,1)$, and in addition $\hat{q} a-\hat{a} \geq 0$.

(a) If $-a \in[0,1]$ and $-b \notin[0,1]$ (see Fig 2 a), as clearly $(x,-a) \in S(0)$, we have 


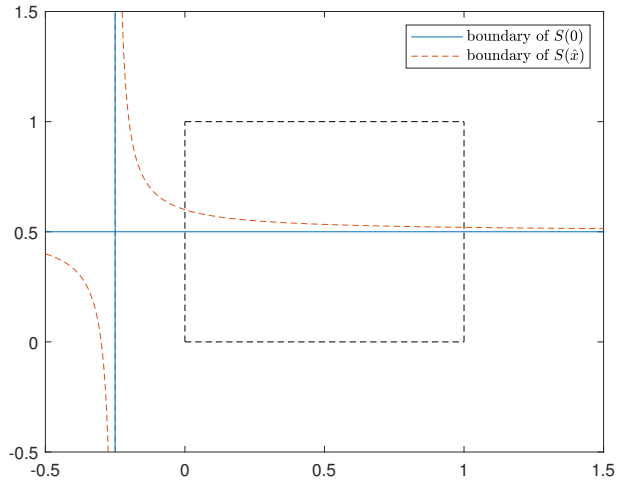

(a) Case (i) (a)

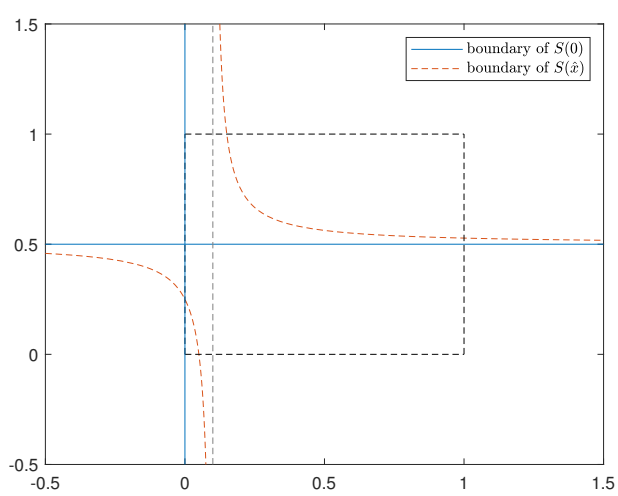

(c) Case (i) (e) 1

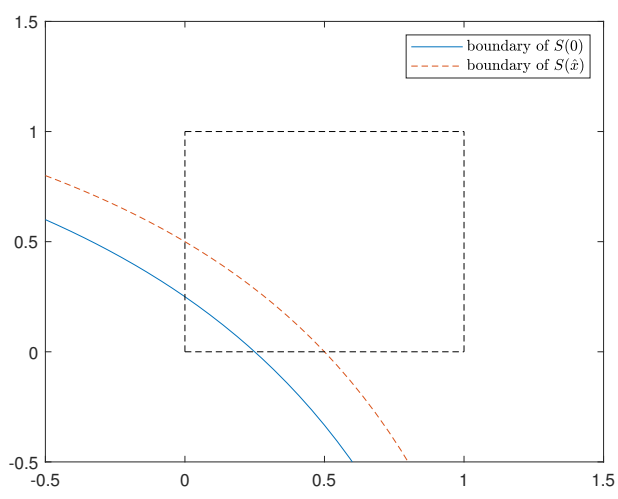

(e) Case (ii) (a)

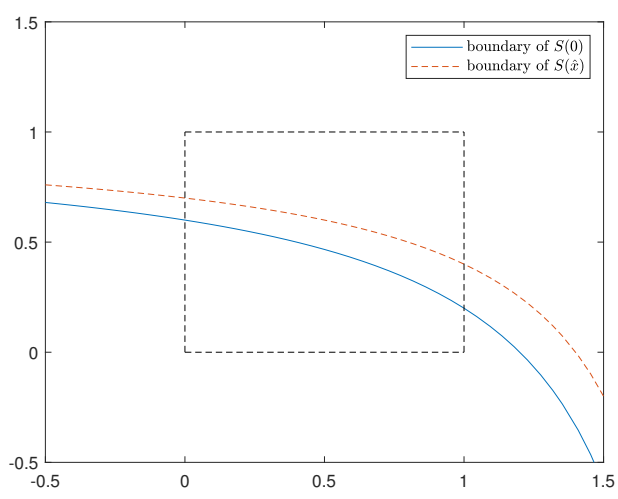

(g) Case (ii)(a) 4

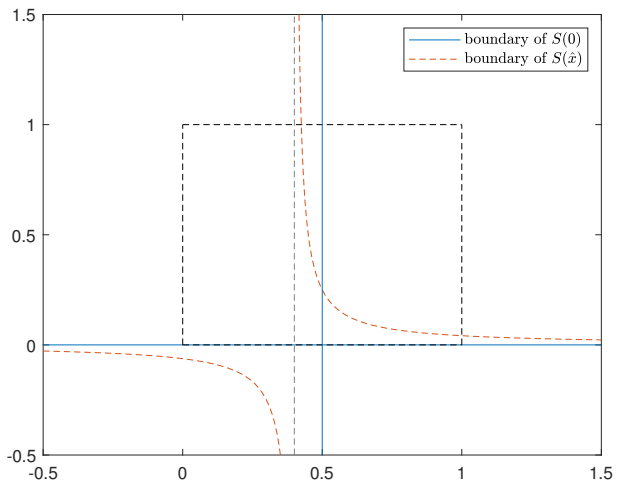

(b) Case (i)|(d) 1

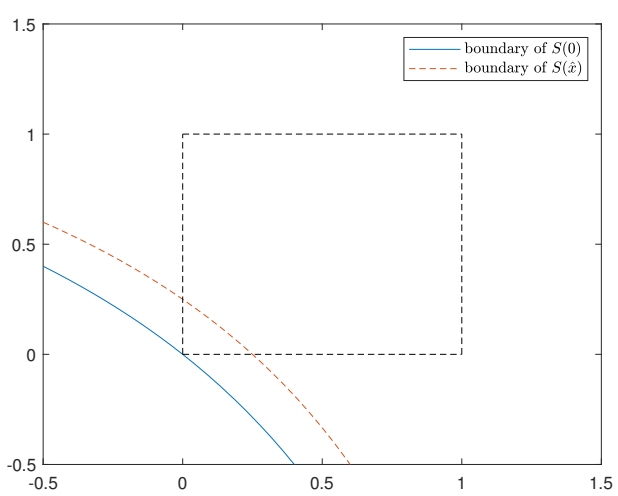

(d) Case (ii) (a)

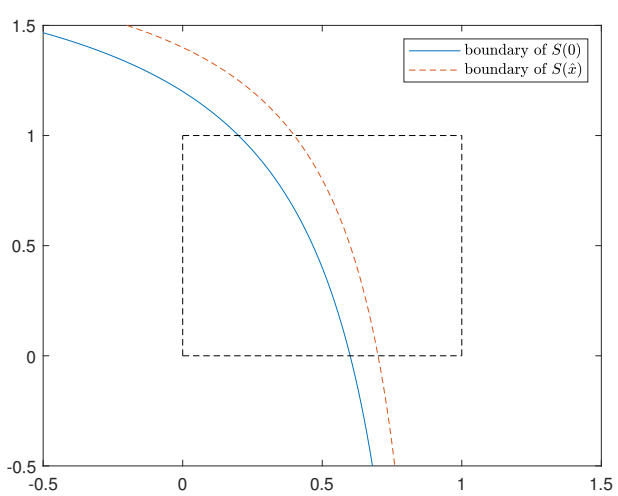

(f) Case (ii)|(a)

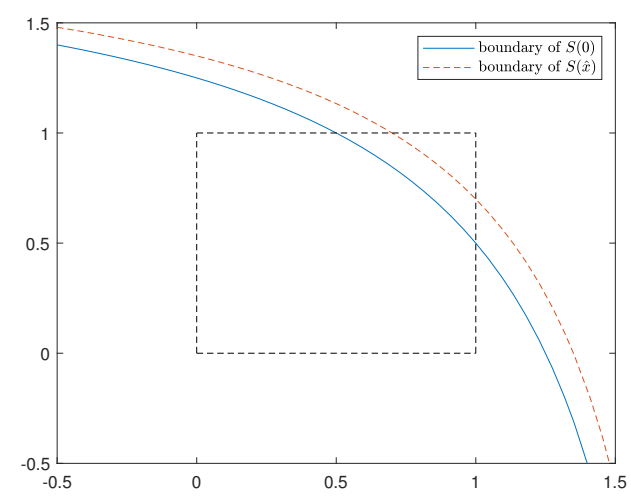

(h) Case (ii) (a) $\bar{p}$ 


$$
\begin{aligned}
& v_{5}(\hat{x})-v(0) \\
\leq & \max \left\{\begin{array}{lll}
\max & r-h(x, y)-v(0) \\
\text { s.t. } \quad(x, y) \in S(0) & \max & \begin{array}{l}
(r-h(x, y))-(r-h(x,-a)) \\
x y+a x+b y+\hat{a} \hat{x}+\hat{q} \hat{x} y=c
\end{array} \\
(x, y) \in[0,1]^{2} \backslash S(0)
\end{array}\right\} \\
\leq & \max \left\{\begin{array}{ll}
\max \quad h(x,-a)-h(x, y) \\
0, & \text { s.t. } \begin{array}{l}
y=\frac{(\hat{q} a-\hat{a}) \hat{x}}{x+b+\hat{x}}-a, \\
(x, y) \in[0,1]^{2} \backslash S(0)
\end{array}
\end{array}\right\} .
\end{aligned}
$$

Thus, depending on the sign of $b$ (i.e. sign of $x+b+\hat{q} \hat{x}$ for sufficiently small $\hat{x}$ ), for $(x, y) \in[0,1]^{2} \backslash S(0)$ we have, using concavity of $h$, either

$$
h(x, y) \geq \frac{\Delta(x, \hat{x})}{1+a} h(x, 1)+\left(1-\frac{\Delta(x, \hat{x})}{1+a}\right) h(x,-a) \geq \frac{\Delta(x, \hat{x})}{1+a}\left(h^{m}-h^{M}\right)+h(x,-a)
$$

or

$$
h(x, y) \geq \frac{-\Delta(x, \hat{x})}{a} h(x, 0)+\left(1-\frac{-\Delta(x, \hat{x})}{a}\right) h(x,-a) \geq \frac{-\Delta(x, \hat{x})}{a}\left(h^{m}-h^{M}\right)+h(x,-a)
$$

where $\Delta(x, \hat{x}):=\frac{(\hat{q} a-\hat{a}) \hat{x}}{x+b+\hat{q} \hat{x}}$ is greater than 0 in the first case and is less than 0 in the second. From the continuity of $h$ we can get $\xi$ independent of $x$ and $\hat{x}$ such that $h(x, y) \geq h(x,-a)+\xi \Delta(x, \hat{x})$.

Therefore, we conclude that

$$
\begin{aligned}
v_{5}(\hat{x})-v(0) & \leq \max \left\{0, \max _{x \in[0,1]}-\xi \Delta(x, \hat{x})\right\}=\max \left\{0, \max _{x \in\{0,1\}}-\xi \Delta(x, \hat{x})\right\} \\
& =\max \left\{0,\left.\max _{x \in\{0,1\}} \frac{-\xi \partial \Delta(x, \hat{x})}{\partial \hat{x}}\right|_{\hat{x}=0} \hat{x}+o(\hat{x})\right\}, \quad(\hat{x} \downarrow 0)
\end{aligned}
$$

where the second equation comes from the monotonicity of $\Delta(x, \hat{x})$ on $x \in[0,1]$ for sufficiently small $\hat{x}$, due to the fact that $-b \notin[0,1]$. Since $x+b \neq 0$, we have that $\left|\max _{x \in\{0,1\}} \frac{-\xi \partial \Delta(x, \hat{x})}{\partial \hat{x}}\right|_{\hat{x}=0} \mid<\infty$. Thus, there exists $l<\infty$ such that $v_{5}(\hat{x})-v(0) \leq l \hat{x}+o(\hat{x})$ for $\hat{x} \downarrow 0$.

(b) If $-b \in[0,1]$ and $-a \notin[0,1]$, a similar analysis can be conducted to obtain $l<\infty$ such that $v_{5}(\hat{x})-v(0) \leq l \hat{x}+o(\hat{x})$ for $\hat{x} \downarrow 0$.

(c) If $-a=-b=0$ or $-a=-b=1$, then $S(0)=[0,1]^{2}$ so that $v_{5}(\hat{x})-v(0) \leq 0$.

(d) (subcase 1) If $-a=0$ with $-b \in(0,1]$ (see Fig 2b), we have $S(0) \supseteq[-b, 1] \times[0,1]$ and $S_{5}(\hat{x}) \subset$ $[-b-\hat{q} \hat{x}, 1] \times[0,1]($ since $(\hat{q} a-\hat{a}) \hat{x} \geq 0)$. Therefore, for $\hat{q} \leq 0, v_{5}(\hat{x})-v(0) \leq 0$ and for $\hat{q}>0$,

$$
\begin{aligned}
& v_{5}(\hat{x})-v(0) \\
\leq & \max \left\{\begin{array}{lll}
\max & r-h(x, y)-v(0) \\
\text { s.t. } \quad(x, y) \in S(0) & \max & (r-h(x, y))-(r-h(-b, y)) \\
& \text { s.t. } & \begin{array}{l}
x y+a x+b y+\hat{a} \hat{x}+\hat{q} \hat{x} y=c \\
(x, y) \in[0,1]^{2} \backslash S(0)
\end{array}
\end{array}\right\} \\
\leq & \max \left\{\begin{array}{lll}
0, & \max \quad h(-b, y)-h(x, y) \\
& \text { s.t. } \quad(x, y) \in[-b-\hat{q} \hat{x},-b] \times[0,1]
\end{array}\right\} .
\end{aligned}
$$

Note that for $x \in[-b-\hat{q} \hat{x},-b]$, using concavity of $h$, we have that

$$
\begin{aligned}
h(x, y) & \geq \frac{x}{-b} h(-b, y)+\frac{-b-x}{-b} h(0, y) \\
& \geq \frac{-b-x}{-b}\left(h^{m}-h^{M}\right)+h(-b, y) \geq \frac{\hat{q} \hat{x}}{-b}\left(h^{m}-h^{M}\right)+h(-b, y) .
\end{aligned}
$$

Thus $v_{5}(\hat{x})-v(0) \leq \max \left\{0, \frac{\hat{q}\left(h^{m}-h^{M}\right)}{b} \hat{x}\right\}$ and we obtain $l<\infty$ such that $v_{5}(\hat{x})-v(0) \leq l \hat{x}$.

(subcase 2) If $-a=1$ with $-b \in[0,1)$, this is the same as (i) (d) 1 as we might perform $x \leftarrow 1-x$ together with $y \leftarrow 1-y$. 
(e) (subcase 1) If $-b=0$ with $-a \in(0,1)$ (see Fig $2 c$, we have $S(0) \supseteq([0,1] \times[-a, 1]) \cup(\{0\} \times[0,1])$ and $S_{5}(\hat{x}) \subset([0,1] \times[-a, 1]) \cup([0,-\hat{q} \hat{x}] \times[0,1])$. For $\hat{q} \geq 0$ we have $S_{5}(\hat{x}) \subset S(0)$ so $v_{5}(\hat{x})-v(0) \leq 0$. For $\hat{q}<0$, we have

$$
\begin{aligned}
& v_{5}(\hat{x})-v(0) \\
& \leq \max \left\{\begin{array}{llll}
\max & r-h(x, y)-v(0) \\
\text { s.t. } & (x, y) \in S(0) & \max & (r-h(x, y))-(r-h(0, y)) \\
\text { s.t. } & \begin{array}{l}
x y+a x+b y+\hat{a} \hat{x}+\hat{q} \hat{x} y=c \\
(x, y) \in[0,1]^{2} \backslash S(0)
\end{array}
\end{array}\right\} \\
& \leq \max \left\{\begin{array}{lll}
0, & \max \quad h(0, y)-h(x, y) \\
\text { s.t. } \quad(x, y) \in[0,-\hat{q} \hat{x}] \times[0,1]
\end{array}\right\} .
\end{aligned}
$$

For $(x, y) \in[0,-\hat{q} \hat{x}] \times[0,1]$, using concavity of $h$, we write

$$
\begin{aligned}
h(x, y) & \geq(1-x) h(0, y)+x h(1, y) & \geq h(0, y)-x(h(0, y)-h(1, y)) \\
& \geq h(0, y)-x\left(h^{M}-h^{m}\right) & \geq h(0, y)+\hat{q} \hat{x}\left(h^{M}-h^{m}\right) .
\end{aligned}
$$

Thus $v_{5}(\hat{x})-v(0) \leq \max \left\{0, \hat{q}\left(h^{m}-h^{M}\right) \hat{x}\right\}$ and we get $l<\infty$ such that $v_{5}(\hat{x})-v(0) \leq l \hat{x}$.

(subcase 2) If $-b=1$ with $-a \in(0,1)$, the argument is the same as for (i) (e) 1 after performing $x \leftarrow 1-x$ and $y \leftarrow 1-y$.

(ii) We next consider the case when $c+a b \neq 0$. As discussed above, we assume $c+a b+(\hat{q} a-\hat{a}) \hat{x} \geq 0$ for all $\hat{x}>0$ and sufficiently small, and thus $c+a b>0$. In addition, if $(x, y) \in S(\hat{x})$ for $\hat{x}>0$ and sufficiently small, we have $x>-b-\hat{q} \hat{x}, y>-a$ or $x<-b-\hat{q} \hat{x}, y<-a$ but not both.

(a) In the case $x<-b-\hat{q} \hat{x}, y<-a$ for $(x, y) \in S(\hat{x})$, we denote $S^{\prime}(0)=S(0) \cap\{(x, y) \mid y<-a, x<$ $-b\} \subseteq S(0)$. Since $(0,0) \in S^{\prime}(0)$, we may assume $(1,1) \notin S^{\prime}(0)$, or $S^{\prime}(0) \supseteq[0,1]^{2}$. Then clearly $S(\hat{x}) \subseteq S(0)$ and therefore $v_{5}(\hat{x}) \leq v(0) \leq 0$.

(Subcase 1) If $S^{\prime}(0)=\{(0,0)\}$ (see Fig 2d), we have $c=0$ and $a, b<0$. Thus, for $\hat{x}$, we obtain a curve between $\left(0,-\frac{\hat{a} \hat{x}}{b+\hat{q} \hat{x}}\right)$ and $\left(-\frac{\hat{a} \hat{x}}{a}, 0\right)$. Thus, for any $(x, y)$ within the curve, from concavity of $h$, it is clear that

$$
\begin{aligned}
h(x, y) \geq & \min \left\{h(0,0), h\left(0,-\frac{\hat{a} \hat{x}}{b+\hat{q} \hat{x}}\right), h\left(-\frac{\hat{a} \hat{x}}{a}, 0\right), h\left(-\frac{\hat{a} \hat{x}}{a},-\frac{\hat{a} \hat{x}}{b+\hat{q} \hat{x}}\right)\right\} \\
\geq & h(0,0)+\min \left\{0,-\frac{\hat{a} \hat{x}}{b+\hat{q} \hat{x}}(h(0,1)-h(0,0)),-\frac{\hat{a} \hat{x}}{a}(h(1,0)-h(0,0)),\right. \\
& \left.-\frac{\hat{a} \hat{x}}{b+\hat{q} \hat{x}}(h(0,1)-h(0,0))-\frac{\hat{a} \hat{x}}{a}(h(1,0)-h(0,0))\right\} \\
\geq & h(0,0)+\max \left\{0,-\frac{\hat{a} \hat{x}}{b+\hat{q} \hat{x}},-\frac{\hat{a} \hat{x}}{a},-\frac{\hat{a} \hat{x}}{b+\hat{q} \hat{x}}-\frac{\hat{a} \hat{x}}{a}\right\}\left(h^{m}-h^{M}\right) \\
= & h(0,0)+\xi \hat{x}+o(\hat{x}), \quad(\hat{x} \downarrow 0)
\end{aligned}
$$

where the second inequality uses the concavity of $h$ and the fact that for sufficiently small $\hat{x},-\frac{\hat{a} \hat{x}}{b+\hat{q} \hat{x}}+$ $\left(-\frac{\hat{a} \hat{x}}{a}\right) \leq 1$, and $\xi$ is a constant obtained by taking the derivative of the "max" term times $h^{m}-h^{M}$ (since $a, b<0$, the term is differentiable.) Thus, by setting $l=\xi$ we have $v_{5}(\hat{x})-v(0) \leq l \hat{x}+o(\hat{x})$ for all sufficiently small $\hat{x}$.

(Subcase 2) If $S^{\prime}(0) \backslash\{(0,0)\} \neq \emptyset$, but $(0,1),(1,0) \notin S^{\prime}(0)$ (see Fig 2e). Then the curve for $\hat{x}=0$ is between $\left(0, \frac{c}{b}\right)$ and $\left(\frac{c}{a}, 0\right)$. We find $\tilde{x}, \tilde{y}$ such that $\left(\tilde{x}, \frac{c}{2 b}\right),\left(\frac{c}{2 a}, \tilde{y}\right)$ are within the curve. From the convex nature of one part of the hyperbola, it can be verified that $\tilde{y}>c / 2 b, \tilde{x}>c / 2 a$. Consider $x \in[0, \tilde{x}]$ and 
denote $y^{\prime}(x)=(c-a x) /(x+b)$, noting that $\left(x, y^{\prime}(x)\right) \in S(0)$ and

$$
\begin{aligned}
& w_{x}(x, \hat{x}):=\max _{y}\left\{(r-h(x, y))-v(0) \mid(x, y) \in S_{5}(\hat{x})\right\}, \\
& \leq \max \left\{\begin{array}{llll}
\max _{y} & (r-h(x, y))-v(0)) & \max _{y} & (r-h(x, y))-\left(r-h\left(x, y^{\prime}\right)\right) \\
\text { s.t. } & (x, y) \in S(0) & \text { s.t. } & x y+a x+b y+\hat{a} \hat{x}+\hat{q} \hat{x} y=c, \\
& & & (x, y) \in[0,1]^{2} \backslash S(0)
\end{array}\right\} \\
& = \begin{cases}\max \left\{0, h\left(x, y^{\prime}(x)\right)-h\left(x, y^{\prime}(x)+\Delta(x, \hat{x})\right)\right\} & \Delta(x, \hat{x}) \geq 0 \\
0 & \Delta(x, \hat{x})<0\end{cases}
\end{aligned}
$$

where

$$
\Delta(x, \hat{x}):=\frac{c-a x-\hat{a} \hat{x}}{x+b+\hat{q} \hat{x}}-\frac{c-a x}{x+b}=\frac{a x \hat{q}-\hat{a} x-\hat{a} b-c \hat{q}}{(x+b+\hat{q} \hat{x})(x+b)} \hat{x}
$$

while noting that if $\Delta(x, \hat{x})<0$, then $\left(x, y^{\prime}(x)+\Delta(x, \hat{x})\right) \in S(0)$.

Note that $[0, \tilde{x}] \subseteq \operatorname{Proj}_{x} S^{\prime}(0)$ and thus $x+b<0$ for $x \in[0, \tilde{x}]$ and therefore for $0<\hat{x}<\hat{x}_{0}$ sufficiently small, we obtain $\frac{\hat{q} \hat{x}}{x+b} \geq-\frac{1}{2}$ or $2 \frac{x+b+\hat{q} \hat{x}}{x+b} \geq 1$ for any $x \in[0, \tilde{x}]$. Thus for $\Delta(x, \hat{x}) \geq 0$,

$$
\Delta(x, \hat{x}) \leq 2 \frac{a x \hat{q}-\hat{a} x-\hat{a} b-c \hat{q}}{(x+b)^{2}} \hat{x} \leq \max _{x \in[0, \tilde{x}]}\left\{2 \frac{a x \hat{q}-\hat{a} x-\hat{a} b-c \hat{q}}{(x+b)^{2}}\right\} \hat{x}:=l_{5, x}^{\prime} \hat{x}
$$

while noting that continuity gives $l_{5, x}^{\prime}<\infty$ independent of $x, \hat{x}$.

Now for the case of $\Delta(x, \hat{x}) \geq 0$ we have that

$$
\begin{aligned}
& h\left(x, y^{\prime}(x)\right)-h\left(x, y^{\prime}(x)+\Delta(x, \hat{x})\right) \\
\leq & \frac{\Delta(x, \hat{x})}{1-y^{\prime}(x)}\left(h\left(x, y^{\prime}(x)\right)-h(x, 1)\right) \leq \frac{\Delta(x, \hat{x})}{1-y^{\prime}(x)}\left(h^{M}-h^{m}\right) \\
\leq & \left(h^{M}-h^{m}\right) \max _{x \in[0, \tilde{x}]}\left\{\frac{1}{1-y^{\prime}(x)}\right\} \max _{x \in[0, \tilde{x}]} \Delta(x, \hat{x}) \\
\leq & \left(h^{M}-h^{m}\right) \max _{x \in[0, \tilde{x}]}\left\{\frac{1}{1-y^{\prime}(x)}\right\} l_{5, x}^{\prime} \hat{x},
\end{aligned}
$$

Therefore, there exists $l_{5, x}<\infty$ independent of $x, \hat{x}$ such that $w_{x}(x, \hat{x}) \leq l_{5, x} \hat{x}+o(\hat{x})$.

A similar analysis of $w_{y}(y, \hat{x}):=\max _{x}\{r-h(x, y)-v(0) \mid x, y \in S(0)\}$ provides $w_{y}(y, \hat{x}) \leq l_{5, y} \hat{x}+o(\hat{x})$ for $\hat{x} \downarrow 0$, where $l_{5, y}$ is a constant independent of $y \in[0, \tilde{y}]$ and $\hat{x}$.

Finally, we combine the results for $w_{x}$ and $w_{y}$. Since they cover the whole curve with overlapping, we have $v_{5}(\hat{x})-v(0) \leq \max \left\{l_{5, x}, l_{5, y}\right\} \hat{x}+o(\hat{x})$ for $\hat{x} \downarrow 0$.

(Subcase 3) If $(0,1) \in S^{\prime}(0)$ but $(1,0) \notin S^{\prime}(0)$ (see Fig 2f), we apply a similar analysis for $w_{y}(y, \hat{x})$ with $y \in[0,1]$ and obtain a constant $l_{5, y}$ independent of $y$ and $\hat{x}$. We thus get $v_{5}(\hat{x})-v(0) \leq l_{5, y} \hat{x}+o(\hat{x})$ for $\hat{x} \downarrow 0$.

(Subcase 4) If $(1,0) \in S^{\prime}(0)$ but $(0,1) \notin S^{\prime}(0)$ (see Fig $2 \mathrm{~g}$ ), we apply a similar analysis for $w_{x}(x, \hat{x})$ with $x \in[0,1]$ and similarly obtain a constant $l_{5, x}$ independent of $x$ and $\hat{x}$.

(Subcase 5) If $(1,0),(0,1) \in S^{\prime}(0)$ (see Fig $2 \mathrm{~h}$ ). Then the curve for $\hat{x}=0$ is between $\left(1, \frac{c-a}{1+b}\right)$ and $\left(\frac{c-b}{1+a}, 1\right)$. Similar to (ii) (a) 2 , we find $\tilde{x}, \tilde{y}$ such that $\left(\tilde{x}, \frac{c-a+b+1}{2+2 b}\right),\left(\frac{c-b+a+1}{2+2 a}, \tilde{y}\right)$ are within the curve. From the convex nature of one part of the hyperbola, we have $\tilde{y}>\frac{c-b+a+1}{2+2 a}$ and $\tilde{x}>\frac{c-a+b+1}{2+2 b}$. Similar to (ii) (a) 2 , we consider $w_{x}(x, \hat{x})$ for $x \in\left[\frac{c-a+b+1}{2+2 b}, 1\right]$ and $w_{y}(y, \hat{x})$ for $y \in\left[\frac{c-b+a+1}{2+2 a}, 1\right]$. We obtain $l_{5, x}$ and $l_{5, y}$. Therefore, we write $v_{5}(\hat{x})-v(0) \leq \max \left\{l_{5, x}, l_{5, y}\right\} \hat{x}+o(\hat{x})$ for $\hat{x} \downarrow 0$.

(b) If for $\hat{x} \in\left(0, \hat{x}_{0}\right)$, and for $(x, y) \in S(\hat{x}) x>-b-\hat{q} \hat{x}, y>-a$, we denote $S^{\prime}(0)=S(0) \cap\{y>-a, x>$ $-b\} \subseteq S(0)$. If $(1,1) \in S^{\prime}(0)$ while $(0,0) \notin S^{\prime}(0)$, the proof is the same as (ii)(a) as we can perform $x \leftarrow 1-x$ with $y \leftarrow 1-y$.

Combining the discussions for (i) and (ii) shows that there exists $l_{5}<\infty$ such that $v_{5}(\hat{x})-v(0) \leq l_{5} \hat{x}+o(\hat{x})$ for $\hat{x} \downarrow 0$. 


\section{Proof of Proposition 1}

Proposition 1. Consider any feasible solution $\left(x^{\prime}, y^{\prime}\right) \in Q$. Then

$$
\begin{aligned}
& \sum_{i \in J_{0} \cup J_{1}} \gamma_{i}\left(x_{i}^{\prime}, y_{i}^{\prime}\right) \geq \sum_{i \in J_{0}} \psi\left(a_{i} x_{i}^{\prime} y_{i}^{\prime}\right)+\sum_{i \in J_{1}} \psi\left(a_{i} x_{i}^{\prime} y_{i}^{\prime}-a_{i}\right) \\
\geq & \psi\left(\sum_{i \in J_{0}} a_{i} x_{i}^{\prime} y_{i}^{\prime}+\sum_{i \in J_{1}}\left(a_{i} x_{i}^{\prime} y_{i}^{\prime}-a_{i}\right)\right) \geq \phi\left(\sum_{i \in J_{0}} a_{i} x_{i}^{\prime} y_{i}^{\prime}+\sum_{i \in J_{1}} a_{i} x_{i}^{\prime} y_{i}^{\prime}-\sum_{i \in J_{1}} a_{i}\right) \\
= & \max _{\left(x_{I}, y_{I}\right) \in[0,1]^{2|I|}}\left\{r-h\left(x_{I}, y_{I}\right) \mid \sum_{i \in I} a_{i} x_{i} y_{i} \geq d-\sum_{i \in J_{0} \cup J_{1}} a_{i} x_{i}^{\prime} y_{i}^{\prime}\right\} \geq r-h\left(x_{I}^{\prime}, y_{I}^{\prime}\right),
\end{aligned}
$$

where the first inequality holds because of assumptions (iii) and (iv) the second inequality holds because assumption (ii) requires $\psi(\cdot)$ to be subadditive over its range, the third inequality holds because assumption (i) requires $\psi(\cdot)$ to be an upper bound on $\phi(\cdot)$, the equality holds from the definition of $\phi(\cdot)$, and the last inequality is satisfied because $\left(x_{I}^{\prime}, y_{I}^{\prime}\right)$ is a feasible solution to the preceding optimization problem.

\section{Proof of Theorem 2}

Theorem [. Suppose $Q \neq \emptyset$. It follows from [23] that the extreme points of $Q$ are such that $\left(x_{j}^{*}, y_{j}^{*}\right) \in\{0,1\}^{2}$ for all $j \in[n] \backslash\{i\}$ for some $i \in[n]$.

Assume first that $Q$ has an extreme point $\left(x^{*}, y^{*}\right)$ where $x_{i}^{*} y_{i}^{*} \notin\{0,1\}$ for some $i \in[n]$ with $a_{i}>0$. Define the partition $\Lambda$ with $I=\{i\}, J_{0}=\left\{j \mid x_{j}^{*} y_{j}^{*}=0\right\}$, and $J_{1}=\left\{j \mid x_{j}^{*} y_{j}^{*}=1\right\}$. Since $\sum_{j=1}^{n} a_{j} x_{j}^{*} y_{j}^{*}=d$, we have $a_{i}>a_{i} x_{i}^{*} y_{i}^{*}=d-\sum_{i \in J_{1}} a_{j}=d^{\Lambda}>0$. Since $d^{\Lambda}>0$, we conclude that $\Lambda$ is a minimal cover yielding partition.

Assume second that all extreme points $\left(x^{*}, y^{*}\right)$ are such that $\left(x_{i}^{*}, y_{i}^{*}\right) \in\{0,1\}^{2}$ for all $i \in[n]$ with $a_{i}>0$. Denote $I_{+}=\left\{i \in[n] \mid a_{i}>0\right\}$ and, for $K_{1}, K_{2} \subseteq I_{+}$, define

$$
Q_{K_{1}, K_{2}}:=\operatorname{conv}\left\{\begin{array}{l|l}
(x, y) \in[0,1]^{2 n} & \begin{array}{l}
\sum_{i=1}^{n} a_{i} x_{i} y_{i} \geq d \\
x_{i}=0, i \in K_{1} \\
y_{i}=0, i \in K_{2} \\
x_{i}=1, i \in I_{+} \backslash K_{1} \\
y_{i}=1, i \in I_{+} \backslash K_{2}
\end{array}
\end{array}\right\} .
$$

It is clear that $\operatorname{conv}(Q)=\operatorname{conv}\left(\bigcup_{K_{1}, K_{2} \subseteq I_{+}} Q_{K_{1}, K_{2}}\right)$. Because, for any $K_{1}, K_{2} \subseteq I_{+}, Q_{K_{1}, K_{2}}$ is a polytope [51, Proposition 17], we conclude that $\operatorname{conv}(Q)$ is a polytope.

\section{Proof of Theorem 3}

Theorem [3. For $i \in[n]$, define

$$
Q_{i}=\left\{\begin{array}{l|l}
(x, y) \in[0,1]^{2 n} & \begin{array}{l}
\left(x_{j}, y_{j}\right)=(1,1), \forall j \in[n] \backslash i \\
\sqrt{a_{i}} \sqrt{x_{i} y_{i}} \geq \sqrt{d_{i}}
\end{array}
\end{array}\right\} .
$$

First observe that, because $a_{i}$ for $i \in[n]$ form a minimal cover, we have that $a_{i}>d_{i}:=d-\sum_{j \neq i} a_{j}$ for each $i$. This implies that sets $Q_{i}$ are nonempty. We next $\operatorname{argue}$ that $\operatorname{conv}(Q)=\operatorname{conv}(\bar{Q})$ where $\bar{Q}:=\bigcup_{i=1}^{n} Q_{i}$. To this end, consider any extreme point $(x, y)$ of $Q$. Then, [23] shows that there exists a partition $\left(I_{0}, I_{1},\{i\}\right)$ of $[n]$ such that $x_{j} y_{j}=0$ for $j \in I_{0}, x_{j} y_{j}=1$ for $j \in I_{1}$ and $x_{i} y_{i} \in[0,1]$. Because $a_{i}$ for $i \in[n]$ form a minimal cover, it must be that $\left|I_{0}\right|=0$ as otherwise $\sum_{j=1}^{n} a_{j} x_{j} y_{j} \leq \sum_{j \in I_{1}} a_{j}<d$. We conclude that $(x, y) \in Q_{i}$. Since $Q$ is compact, it follows that $\operatorname{conv}(Q) \subseteq \operatorname{conv}(\bar{Q})$. Further, since $Q_{i} \subseteq \bar{Q} \subseteq Q$, it is clear that $\operatorname{conv}(\bar{Q}) \subseteq \operatorname{conv}(Q)$.

We now use disjunctive programming to obtain an extended formulation of $\operatorname{conv}(\bar{Q})$. This formulation introduces convex multipliers $\lambda_{i}$ and copies $\left(x^{i}, y^{i}\right)$ of variables $(x, y)$ for each disjunct $Q_{i}$. Because disjunct $Q_{i}$ 
yields constraints $y_{j}^{i}=x_{j}^{i}=\lambda_{i}$ for $j \neq i$, variables $x_{j}^{i}$ and $y_{j}^{i}$ for $j \neq i$ can be eliminated from the formulation in favor of $\lambda_{i}$. Renaming variables $x_{i}^{i}$ as $\hat{x}_{i}$, we obtain

$$
\begin{aligned}
x_{j} & =\hat{x}_{j}+\sum_{i \neq j} \lambda_{i} & & \forall j \in[n] \\
y_{j} & =\hat{y}_{j}+\sum_{i \neq j} \lambda_{i} & & \forall j \in[n] \\
\sqrt{a_{i}} \sqrt{\hat{x}_{i} \hat{y}_{i}} & \geq \sqrt{d_{i}} \lambda_{i} & & \forall i \in[n] \\
\lambda_{i} & \geq \hat{x}_{i}, \hat{y}_{i} \geq 0 & & \forall i \in[n] \\
\sum_{i=1}^{n} \lambda_{i} & =1 & &
\end{aligned}
$$

because the constraint functions of each $Q_{i}$ are positively homogeneous.

Using the fact that $\sum_{i \neq j} \lambda_{i}=1-\lambda_{j}$, we obtain $\hat{x}_{j}=x_{j}-\left(1-\lambda_{j}\right)$ and $\hat{y}_{j}=y_{j}-\left(1-\lambda_{j}\right)$. Eliminating these variables from the formulation, we obtain

$$
\begin{aligned}
\sqrt{a_{i}} \sqrt{\left(x_{i}-\left(1-\lambda_{i}\right)\right) \cdot\left(y_{i}-\left(1-\lambda_{i}\right)\right)} & \geq \sqrt{d_{i}} \lambda_{i} & \forall i \in[n] \\
1-\lambda_{i} \leq x_{i}, y_{i} & \leq 1 & \forall i \in[n] \\
\sum_{i=1}^{n} \lambda_{i} & =1 . &
\end{aligned}
$$

Because projecting variables $\lambda_{i}$ from the above formulation seems difficult, we relax the above set by using, for each $i \in[n]$ the following inequality

$$
\sqrt{a_{i}}\left(\sqrt{x_{i} y_{i}}-\left(1-\lambda_{i}\right)\right) \geq \sqrt{a_{i}} \sqrt{\left(x_{i}-\left(1-\lambda_{i}\right)\right) \cdot\left(y_{i}-\left(1-\lambda_{i}\right)\right)},
$$

which holds as $\left(\sqrt{x_{i} y_{i}}-\left(1-\lambda_{i}\right)\right)^{2} \geq x_{i} y_{i}-\left(x_{i}+y_{i}\right)\left(1-\lambda_{i}\right)+\left(1-\lambda_{i}\right)^{2}=\left(x_{i}-\left(1-\lambda_{i}\right)\right)\left(y_{i}-\left(1-\lambda_{i}\right)\right)$ where the first inequality is obtained by expanding the square and using the arithmetic-geometry mean inequality $-2 \sqrt{x_{i} y_{i}} \geq-\left(x_{i}+y_{i}\right)$. Substituting (11) in (10), we obtain:

$$
\begin{aligned}
\lambda_{i} & \geq \frac{\sqrt{a_{i}}}{\sqrt{a_{i}}-\sqrt{d_{i}}}\left(1-\sqrt{x_{i} y_{i}}\right) & & \forall i \in[n] \\
\lambda_{i} & \geq 1-x_{i} & & \forall i \in[n] \\
\lambda_{i} & \geq 1-y_{i} & & \forall i \in[n] \\
x_{i}, y_{i} & \leq 1 & & \forall i \in[n] \\
\sum_{i=1}^{n} \lambda_{i} & =1 . & &
\end{aligned}
$$

Using Fourier-Motzkin to project variables $\lambda_{i}$, we obtain $(x, y) \in[0,1]^{2 n}$ together with

$$
\sum_{i=1}^{n} \max \left\{\frac{\sqrt{a_{i}}}{\sqrt{a_{i}}-\sqrt{d_{i}}}\left(1-\sqrt{x_{i} y_{i}}\right), 1-x_{i}, 1-y_{i}\right\} \leq 1,
$$

which is a convex inequality. Retaining only the first term in the maximum for each pair $\left(x_{i}, y_{i}\right)$ and multiplying through by -1 yields the weaker convex inequality (3).

\section{Proof of Theorem 4}

In this section, we provide a proof of Theorem 4. We say that $G \in \mathbb{R}^{n}$ is a set of the covering type if whenever $\hat{x} \in G$, then $\tilde{x} \in G$ for all $\tilde{x} \in \mathbb{R}^{n}$ such that $\tilde{x} \geq \hat{x}$. Due to lack of space we skip the proof of the next proposition; see [17] for a similar result.

Proposition 3. Let $B=[0,1]^{n}$ and let $G$ and $H$ be sets of the covering type, such that $\operatorname{conv}(G \cap B) \subseteq H$. If there exists $\theta \geq 1$, such that for any $c \geq 0, z^{l} \leq z^{*} \leq \theta z^{l}$, where $z^{*}:=\min \left\{c^{\top} x \mid x \in G \cap B\right\}$ and $z^{l}:=$ $\min \left\{c^{\top} x \mid x \in H \cap B\right\}$, then $(\theta H) \cap B \subseteq \operatorname{conv}(G \cap B)$.

Following Proposition 3 Theorem 4 will be proven if, for all $(p, q) \in \mathbb{R}_{+}^{2 n}$,

$$
\begin{aligned}
& z^{*}:=\min \left\{\sum_{i=1}^{n}\left(p_{i} x_{i}+q_{i} y_{i}\right) \mid \sum_{i=1}^{n} a_{i} x_{i} y_{i} \geq d,(x, y) \in[0,1]^{2 n}\right\} \\
& z^{l}:=\min \left\{\sum_{i=1}^{n}\left(p_{i} x_{i}+q_{i} y_{i}\right) \mid \sum_{i=1}^{n} \frac{\sqrt{a_{i}}}{\sqrt{a_{i}}-\sqrt{d_{i}}}\left(\sqrt{x_{i} y_{i}}-1\right) \geq-1,(x, y) \in[0,1]^{2 n}\right\}
\end{aligned}
$$

satisfy $z^{l} \leq z^{*} \leq 4 z^{l}$. To this end, we prove first four ancillary results in Lemmas 14 
Assumption 1. $p_{i} \geq q_{i}, \forall i \in[n]$.

Assumption 1 is without loss of generality as it can always be achieved by renaming variables $x_{i}$ as $y_{i}$, if necessary.

Lemma 1. For $\alpha \in[0,1]$ and $i \in[n]$, define

$$
\theta_{i}(\alpha)=\min \left\{\begin{array}{l|l}
p_{i} x_{i}+q_{i} y_{i} & \begin{array}{l}
\sqrt{x_{i} y_{i}}=\alpha \\
\left(x_{i}, y_{i}\right) \in[0,1]^{2}
\end{array}
\end{array}\right\}
$$

Then, $\theta_{i}(\alpha)=0$ when $p_{i}=0$. Further, when $p_{i}>0$,

$$
\theta_{i}(\alpha)=\left\{\begin{array}{lll}
2 \sqrt{p_{i} q_{i}} \cdot \alpha & \text { when } \quad \alpha \leq \sqrt{\frac{q_{i}}{p_{i}}} \\
p_{i} \cdot \alpha^{2}+q_{i} & \text { when } \quad \alpha \geq \sqrt{\frac{q_{i}}{p_{i}}} .
\end{array}\right.
$$

Proof. When $p_{i}=0$, it follows from Assumption 1 that $q_{i}=0$. The result holds trivially. For $p_{i}>0$, setting $x_{i}=\alpha^{2} / y_{i}$, we write $\theta_{i}(\alpha)=\min \left\{p_{i} \alpha^{2} / y_{i}+q_{i} y_{i} \mid \alpha^{2} \leq y_{i} \leq 1\right\}$, a problem with linear constraints and a convex objective over $\mathbb{R}_{+}$. When $q_{i}=0, y_{i}^{*}=1$ is optimal and the result follows as $x_{i}^{*}=\alpha^{2}$. When $q_{i}>0$, the problem has $y_{i}^{*}=\sqrt{p_{i} / q_{i}} \alpha \geq \alpha \geq \alpha^{2}$ as unique stationary point over $\mathbb{R}_{+}$. We conclude that $\bar{y}_{i}=\min \left\{y_{i}^{*}, 1\right\}$ is optimal for the constrained problem.

Lemma 2. Let $\alpha_{i}^{*}:=\sqrt{\frac{d_{i}}{a_{i}}}$. Then, $z^{*}=\min _{i \in[n]}\left\{\sum_{j \in[n] \backslash\{i\}}\left(p_{j}+q_{j}\right)+\theta_{i}\left(\alpha_{i}^{*}\right)\right\}$.

Proof. Since an optimal solution to the problem defining $z^{*}$ can always be chosen among the extreme points of $Q$ and since the proof of Theorem 3 in Section 7 establishes that extreme points of $Q$ belong to $\bigcup_{i=1}^{n} Q_{i}$, we write that $z^{*}=\min _{i \in[n]} \min \left\{p^{\top} x+q^{\top} y \mid(x, y) \in Q_{i}\right\}$. Points of $Q_{i}$ satisfy $x_{j}=y_{j}=1$ for $j \neq i$ and $a_{i} x_{i} y_{i} \geq d_{i}$. Since $p_{i} \geq q_{i} \geq 0$, it suffices to consider solutions that satisfy $\sqrt{x_{i} y_{i}}=\sqrt{d_{i} / a_{i}}=\alpha_{i}^{*}$ in the above problem, yielding the result.

Rearranging the variables if necessary, assume from now on that $z^{*}=\sum_{i \in[n-1]}\left(p_{i}+q_{i}\right)+\theta_{n}\left(\alpha_{n}^{*}\right)$. As a consequence of this assumption and Lemma 2, we obtain that

$$
\theta_{j}\left(\alpha_{j}^{*}\right)+p_{n}+q_{n} \geq \theta_{n}\left(\alpha_{n}^{*}\right)+p_{j}+q_{j}, \quad \forall j \in[n] .
$$

Lemma 3. Let $\tau_{i}(\alpha)=\left(p_{i}+q_{i}\right) \cdot \alpha^{2}$. Then $\tau_{i}(\alpha) \leq \theta_{i}(\alpha)$ for $\alpha \in[0,1]$.

Proof. When $p_{i}=0$, the result is clear. Assume therefore that $p_{i}>0$. When $\alpha \geq \sqrt{q_{i} / p_{i}}$, we write that $\theta_{i}(\alpha)=p_{i} \alpha^{2}+q_{i} \geq p_{i} \alpha^{2}+q_{i} \alpha^{2}=\tau_{i}(\alpha)$, where the inequality holds because $\alpha \in[0,1]$. When $\alpha \leq \sqrt{q_{i} / p_{i}}$ (or equivalently $\sqrt{q_{i}} \geq \sqrt{p_{i}} \alpha$, we write that $\theta_{i}(\alpha)=2 \sqrt{q_{i}} \sqrt{p_{i}} \alpha \geq 2 p_{i} \alpha^{2} \geq\left(p_{i}+q_{i}\right) \alpha^{2}$, where the last inequality holds because $p_{i} \geq q_{i} \geq 0$.

Lemma 4. Assume that $(x, y) \in[0,1]^{2 n}$ satisfies 3 3), i.e., $\sum_{i=1}^{n} \frac{\sqrt{a_{i}}}{\sqrt{a_{i}}-\sqrt{d_{i}}}\left(\sqrt{x_{i} y_{i}}-1\right) \geq-1$. Define $\alpha_{i}=\sqrt{x_{i} y_{i}}$ for $i \in[n]$. Then (i) $\alpha_{i}^{*} \leq \alpha_{i}$ for all $i \in[n]$, (ii) $\alpha_{i}<\frac{1}{2}$ for at most one $i \in[n]$.

Proof. Statement (i) trivially holds, as any $\sqrt{x_{i} y_{i}}<\alpha_{i}^{*}=\sqrt{d_{i} / a_{i}}$ invalidates $(3)$, even if we set $x_{j}=y_{j}=1$ for $j \in[n] \backslash\{i\}$. For (ii), assume by contradiction there exists distinct indices $i_{1}$ and $i_{2}$ in $[n]$ such that $\alpha_{i_{1}} \leq \alpha_{i_{2}}<\frac{1}{2}$. Then $\sum_{i=1}^{n} \frac{\sqrt{a_{i}}}{\sqrt{a_{i}}-\sqrt{d_{i}}}\left(\sqrt{x_{i} y_{i}}-1\right)<\sum_{i \in\left\{i_{1}, i_{2}\right\}} \frac{\sqrt{a_{i}}}{\sqrt{a_{i}}-\sqrt{d_{i}}}\left(-\frac{1}{2}\right) \leq \sum_{i \in\left\{i_{1}, i_{2}\right\}}\left(-\frac{1}{2}\right)=-1$, which violates [3.

We are now ready to give a proof of Theorem 4 that inequality (3) yields strong bounds for optimization problems over $Q$.

Theorem 4. Let $(\tilde{x}, \tilde{y})$ be an optimal solution for the relaxation defining $z^{l}$ and let $\tilde{\alpha}_{i}=\sqrt{\tilde{x}_{i} \tilde{y}_{i}}$. From Lemma 4 it is sufficient to consider the following three cases. 
First assume that $\tilde{\alpha}_{j} \leq \frac{1}{2}$ for some $j<n$. Lemma 4 implies $\tilde{\alpha}_{i} \geq \frac{1}{2}$ for $i \neq j$. Then

$$
\begin{aligned}
4 z^{l} & =4 \sum_{i=1}^{n} \theta_{i}\left(\tilde{\alpha}_{i}\right) \geq 4\left(\sum_{i \in[n] \backslash\{j\}}\left(p_{i}+q_{i}\right) \tilde{\alpha}_{i}^{2}+\theta_{j}\left(\tilde{\alpha}_{j}\right)\right) \\
& \geq 4\left(\sum_{i \in[n] \backslash\{j\}}\left(p_{i}+q_{i}\right) \frac{1}{4}+\theta_{j}\left(\tilde{\alpha}_{j}\right)\right)=\sum_{i \in[n] \backslash\{j\}}\left(p_{i}+q_{i}\right)+4 \theta_{j}\left(\tilde{\alpha}_{j}\right) \\
& \geq \sum_{i \in[n] \backslash\{j, n\}}\left(p_{i}+q_{i}\right)+\theta_{j}\left(\alpha_{j}^{*}\right)+\left(p_{n}+q_{n}\right) \\
& \geq \sum_{i \in[n] \backslash\{j, n\}}\left(p_{i}+q_{i}\right)+p_{j}+q_{j}+\theta_{n}\left(\alpha_{n}^{*}\right)=z^{*},
\end{aligned}
$$

where the first inequality holds because of Lemma 3 , the second inequality holds because $\alpha_{i} \geq \frac{1}{2}$ for $i \neq j$, the third inequality is because $\alpha_{i}^{*} \leq \tilde{\alpha}_{j}$ from Lemma 4 and because $\theta_{j}$ is monotonically increasing, and the fourth inequality holds because of 13 .

Second assume that $\tilde{\alpha}_{n} \leq \frac{1}{2}$. Lemma 4 implies that $\tilde{\alpha}_{i} \geq \frac{1}{2}$ for $i<n$. Similarly, $4 z^{l}=4 \sum_{i=1}^{n} \theta_{i}\left(\tilde{\alpha}_{i}\right) \geq$ $4\left(\sum_{i=1}^{n-1}\left(p_{i}+q_{i}\right) \tilde{\alpha}_{i}^{2}+\theta_{n}\left(\tilde{\alpha}_{n}\right)\right) \geq 4\left(\sum_{i=1}^{n-1}\left(p_{i}+q_{i}\right) \frac{1}{4}+\theta_{n}\left(\tilde{\alpha}_{n}\right)\right)=\sum_{i=1}^{n-1}\left(p_{i}+q_{i}\right)+4 \theta_{n}\left(\tilde{\alpha}_{n}\right) \geq \sum_{i=1}^{n-1}\left(p_{i}+q_{i}\right)+\theta_{n}\left(\alpha_{n}^{*}\right)=$ $z^{*}$.

Finally assume that $\tilde{\alpha}_{i} \geq \frac{1}{2}$ for all $i$, and we use the same proof as just given.

\section{Proof of Theorem 5}

In this section, we provide a proof of Theorem 5, which gives a subadditive over-approximation to the lifting function of the minimal covering inequality. We first pose

Assumption 2. $0<\Delta \leq a_{1} \leq a_{2} \leq \ldots \leq a_{n}$.

Assumption 2 can always be achieved by reordering the variables since the notion of minimal cover requires that $a_{i} \geq \Delta$ for $i \in[n]$; see discussion following Notation 1 .

We next present ancillary results in Lemmas 59 and Proposition 4 that are used in the derivation of the approximation of the lifting function. The proof of Lemma 5 is straightforward and can be obtained by investigating signs of derivatives.

Lemma 5. For $u \geq \max \{\alpha, \beta\}$ where $\alpha, \beta>0$, the function $f(u):=\frac{\sqrt{u}-\sqrt{u-\alpha}}{\sqrt{u}-\sqrt{u-\beta}}$ is decreasing when $\alpha>\beta$ and increasing when $\alpha<\beta$.

Lemma 6 establishes that the lifting function $\phi(\delta)$ exhibits local convexity.

Lemma 6. Any point $\delta$ of the lifting function $\phi(\delta)$ corresponding to an optimal solution $(x, y)$ with at least one index $i$ such that $x_{i} y_{i} \in(0,1)$, is locally convex, i.e., there exists $r>0$ and $\xi$ such that $\phi(\delta+\eta) \geq \phi(\delta)+\xi \eta$ for all $\eta \in[-r, r]$.

Proof. Let $\dot{\delta}$ be a point for which an optimal solution $(\dot{x}, \dot{y})$ to the problem defining $\phi(\dot{\delta})$ is such that $\dot{x}_{i} \dot{y}_{i} \in(0,1)$. Define $r=\min \left\{\dot{x}_{i} \dot{y}_{i}, 1-\dot{x}_{i} \dot{y}_{i}\right\} / 2>0$. Consider $\eta \in[-r, r]$ and construct $(x, y)$ so that $x_{j}=\dot{x}_{j}, y_{j}=\dot{y}_{j}$ for any $j \neq i$ and $x_{i} y_{i}=\dot{x}_{i} \dot{y}_{i}-\eta$. From the feasibility of $(\dot{x}, \dot{y})$ for $\dot{\delta}$, we conclude that $(x, y)$ is a feasible solution to the optimization problem defining $\phi(\dot{\delta}+\eta)$. Therefore,

$$
\begin{aligned}
\phi(\dot{\delta}+\eta)-\phi(\dot{\delta}) & \geq \frac{\sqrt{a_{i}}}{\sqrt{a_{i}}-\sqrt{d_{i}}}\left(\sqrt{\dot{x}_{i} \dot{y}_{i}}-\sqrt{\dot{x}_{i} \dot{y}_{i}-\eta}\right) \\
& =\frac{a_{i}+\sqrt{a_{i} d_{i}}}{a_{i}-d_{i}}\left(\sqrt{\dot{x}_{i} \dot{y}_{i}}-\sqrt{\dot{x}_{i} \dot{y}_{i}-\eta}\right) \geq \frac{a_{i}+\sqrt{a_{i} d_{i}}}{2 \Delta \sqrt{\dot{x}_{i} \dot{y}_{i}}} \eta,
\end{aligned}
$$

where the last inequality holds because $a_{i}-d_{i}=\Delta$ and because the concavity of the square root function over $\mathbb{R}_{+}$implies that $\sqrt{\dot{x}_{i} \dot{y}_{i}-\eta} \leq \sqrt{\dot{x}_{i} \dot{y}_{i}}-\frac{1}{2 \sqrt{\dot{x}_{i} \dot{y}_{i}}} \eta$. 
To obtain the tightest linear over-approximation of $\phi(\delta)$ for $\delta \in(0, \infty)$, we next narrow down the set of points $\delta$ where function $\phi(\delta) / \delta$ can achieve a local maximum.

Proposition 4. Assume that $\dot{\delta}>0$ is a local maximizer of the function $\phi(\delta) / \delta$ and that $(\dot{x}, \dot{y})$ is an optimal solution to the problem defining $\phi(\dot{\delta})$. Then either

(i) all $\left(\dot{x}_{i}, \dot{y}_{i}\right)$ pairs belong to $\{0,1\}^{2}$, or

(ii) there exists $r>0$ such that $\phi(\delta+\eta) /(\delta+\eta)=\phi(\delta) / \delta$ for all $\eta \in(-r, r)$.

Proof. Assume that (i) does not hold, i.e., there exists $i \in[n]$ for which $\dot{x}_{i} \dot{y}_{i} \in(0,1)$. We show that (ii) holds. From Lemma 6 , there exists $\xi$ and $r>0$ such that $\phi(\dot{\delta}+\eta) \geq \phi(\dot{\delta})+\xi \eta$ for $\eta \in(-r, r)$. Without loss of generality, we assume $r<\dot{\delta}$. We consider two cases. Assume first that $\xi \geq \phi(\dot{\delta}) / \dot{\delta}$. For any $\eta \in(0, r)$ we have $\phi(\dot{\delta}+\eta) \geq \phi(\dot{\delta})+\xi \eta \geq \frac{\phi(\dot{\delta})}{\dot{\delta}}(\dot{\delta}+\eta)$ or equivalently $\phi(\dot{\delta}+\eta) /(\dot{\delta}+\eta) \geq \phi(\dot{\delta}) / \dot{\delta}$. Assume second that $\xi \leq \phi(\dot{\delta}) / \dot{\delta}$. For any $\eta \in(-r, 0)$ we have $\phi(\dot{\delta}+\eta) \geq \phi(\dot{\delta})+\xi \eta \geq \frac{\phi(\dot{\delta})}{\dot{\delta}}(\dot{\delta}+\eta)$ or equivalently $\phi(\dot{\delta}+\eta) /(\dot{\delta}+\eta) \geq \phi(\dot{\delta}) / \dot{\delta}$. From analyzing these cases, we see that $\dot{\delta}$ can be a local maximum only if $\eta=\phi(\dot{\delta}) / \dot{\delta}$ and all points in $(\delta-r, \delta+r)$ are also local maxima.

We now derive a linear over-approximation to the function $\phi(\delta)$ for $\delta \geq 0$.

Lemma 7. Define $l_{+}:=\frac{\sqrt{a_{i_{0}}}+\sqrt{d_{i_{0}}}}{\Delta \sqrt{d_{i_{0}}}}$ if $I^{>} \neq \emptyset$ and $l_{+}:=\frac{1}{\Delta}$ otherwise. Then $\phi(\delta) \leq l_{+} \delta$ for $\delta \geq 0$.

Proof. The result holds trivially for $\delta=0$ since $\phi(\delta)=0$. Our main tool to prove this result is Proposition 4 which will allow us to verify the value of $\phi(\delta) / \delta$ only for a finite set of values of $\delta$. However, since Proposition 4 holds only for $\delta>0$, we first prove the result in an interval that has 0 as an end point.

As mentioned above, the first part of the proof investigates the function $\phi$ in a neighborhood of the point $\delta=0$. There are two cases to consider.

For the first case, assume that $I^{>} \neq \emptyset$. Consider $\delta \in\left[0, \min \left\{a_{i_{0}}-\Delta, \Delta\right\} / 2\right]$. Because the problem defining $\phi$ consists of maximizing a convex function, optimal solutions can be found at extreme points of the feasible region. It follows that there exists an optimal solution that is such that $x_{i}^{*} y_{i}^{*} \in\{0,1\}$ for all $i \in[n] \backslash\{j\}$ for some $j \in[n]$.

Further, at most one index $k \in[n] \backslash\{j\}$ can be such that $x_{k}^{*} y_{k}^{*}=0$ as otherwise $\sum_{i=1}^{n} a_{i} x_{i}^{*} y_{i}^{*} \leq \sum_{i=1}^{n} a_{i}-2 \Delta=$ $d-\Delta<d-\delta$ which would made this solution infeasible for the problem defining $\phi$.

Also, if there exists $k$ with $x_{k}^{*} y_{k}^{*}=0$, then $a_{k}=\Delta$. If not, $a_{k} \geq a_{i_{0}}$ and thus $\sum a_{i} x_{i}^{*} y_{i}^{*} \leq d+\Delta-a_{i_{0}}<$ $d-\delta$, infeasible. Thus, $-\Delta-\delta=\sum_{i=1}^{n} a_{i} x_{i}^{*} y_{i}^{*}-\sum_{i=1}^{n} a_{i}=a_{j} x_{j}^{*} y_{j}^{*}-a_{j}-a_{k}, i . e ., a_{j} x_{j}^{*} y_{j}^{*}=a_{j}-\delta$, and as $\frac{\sqrt{a_{k}}}{\sqrt{a_{k}}-\sqrt{d_{k}}}=\frac{\sqrt{\Delta}}{\sqrt{\Delta}-0}=1$, we obtain

$$
\phi(\delta)=\frac{\sqrt{a_{j}}}{\sqrt{a_{j}}-\sqrt{d_{j}}}\left(1-\sqrt{x_{j}^{*} y_{j}^{*}}\right)=\frac{\sqrt{a_{j}}-\sqrt{a_{j}-\delta}}{\sqrt{a_{j}}-\sqrt{a_{j}-\Delta}} \leq \frac{\sqrt{a_{n}}-\sqrt{a_{n}-\delta}}{\sqrt{a_{n}}-\sqrt{a_{n}-\Delta}}:=\eta(\delta),
$$

where the last step follows from Lemma 5. Note that $\eta$ is well-defined and convex on $[0, \Delta]$. Therefore, it is easy to verify that $\eta(\delta) \leq \delta / \Delta$ for $\delta \in[0, \Delta]$, and thus $\phi(\delta) \leq \delta / \Delta \leq l_{+} \delta$ for $\delta \in\left[0, \min \left\{a_{i_{0}}-\Delta, \Delta\right\} / 2\right]$.

If there is no $k$ with $x_{k}^{*} y_{k}^{*}=0$, we can verify $a_{j}>\Delta$ and $a_{j} x_{j}^{*} y_{j}^{*}=a_{j}-\Delta-\delta$. Thus,

$$
\begin{aligned}
\phi(\delta) & =\frac{\sqrt{a_{j}}}{\sqrt{a_{j}}-\sqrt{d_{j}}}\left(1-\sqrt{x_{j}^{*} y_{j}^{*}}\right)-1 \\
& =\frac{\sqrt{a_{j}}-\sqrt{a_{j}-\Delta-\delta}}{\sqrt{a_{j}}-\sqrt{a_{j}-\Delta}}-1 \leq \frac{\sqrt{a_{i_{0}}}-\sqrt{a_{i_{0}}-\Delta-\delta}}{\sqrt{a_{i_{0}}}-\sqrt{a_{i_{0}}-\Delta}}-1:=\xi(\delta),
\end{aligned}
$$

where the last step follows Lemma 5 . The function $\xi(\delta)$ is again convex. Therefore, it is easy to verify that $\xi(\delta) \leq \frac{\sqrt{a_{i_{0}}}+\sqrt{d_{i_{0}}}}{\Delta \sqrt{d_{i_{0}}}} \delta$ for $\delta \in\left[0, a_{i_{0}}-\Delta\right]$. Thus we obtain that $\phi(\delta) \leq l_{+} \delta$ for $\delta \in\left[0, \min \left\{a_{i_{0}}-\Delta, \Delta\right\} / 2\right]$.

For the second case $I^{>}=\emptyset$, i.e., $a_{1}=\ldots=a_{n}=\Delta$. Note that in this case $n \geq 2$. Consider $\delta \in[0, \Delta / 2]$. Similar to above, there exists an optimal solution that is such that $x_{i}^{*} y_{i}^{*} \in\{0,1\}$ for all $i \in[n] \backslash\{j\}$ for some 
$j \in[n]$. In addition, there exists exactly one index $k \in[n] \backslash\{j\}$ with $x_{k}^{*} y_{k}^{*}=0$, or otherwise we obtain $a_{j}>\Delta$ a contradiction to $I^{>}=\emptyset$. As $\frac{\sqrt{a_{k}}}{\sqrt{a_{k}}-\sqrt{d_{k}}}=1$ and $a_{j} x_{j}^{*} y_{j}^{*}=\Delta x_{j}^{*} y_{j}^{*}=\Delta-\delta$, we obtain

$$
\phi(\delta)=\frac{\sqrt{a_{j}}}{\sqrt{a_{j}}-\sqrt{d_{j}}}\left(1-\sqrt{x_{j}^{*} y_{j}^{*}}\right)=\frac{\sqrt{\Delta}-\sqrt{\Delta-\delta}}{\sqrt{\Delta}} \leq \frac{\delta}{\Delta} \leq l_{+} \delta, \delta \in[0, \Delta / 2] .
$$

The second part of the proof investigates the function $\phi$ away from the origin. As we are attempting to show that $\phi(\delta) / \delta$ bounded from above by $l_{+}$, it is sufficient to consider all local maximas of $\phi(\delta) / \delta$. It follows from Proposition 4 that it is sufficient to verify the condition at values of $\delta$ such that $x_{i} y_{i} \in\{0,1\}$ for $i \in[n]$. (This is because, at other local maximas, the function $\phi(\delta) / \delta$ is locally constant and so it is sufficient to check at the end points of these "constant intervals" where $x_{i} y_{i} \in\{0,1\}$.) Any such local maximum $\delta$ is therefore such that there exists $S \subseteq[n]$ with $x_{i} y_{i}=0$ for $i \in S$ and $x_{i} y_{i}=1$ for $i \notin S$. We denote it as $\delta^{S}$. It is easily verified that $\delta^{S}=\sum_{i \in S} a_{i}-\Delta$. Let $S=\left\{i_{1}, i_{2}, \ldots, i_{k}\right\}$ such that $a_{i_{1}} \leq \ldots \leq a_{i_{k}}$, and we have

$$
\phi\left(\delta^{S}\right)=-1+\sum_{i \in S} \frac{a_{i}+\sqrt{a_{i} d_{i}}}{\Delta}=\sum_{j=1}^{k-1} \frac{a_{i_{j}}+\sqrt{a_{i_{j}} d_{i_{j}}}}{\Delta}+\frac{d_{i_{k}}+\sqrt{a_{i_{k}} d_{i_{k}}}}{\Delta} .
$$

Consider two cases. On the one hand, if $a_{i_{k}}>\Delta$, then $I^{>} \neq \emptyset$ and $a_{i_{0}} \leq a_{i_{k}}$. Thus,

$$
\begin{aligned}
\phi\left(\delta^{S}\right) & =\sum_{j=1}^{k-1} \frac{\sqrt{a_{i_{j}}}+\sqrt{d_{i_{j}}}}{\Delta \sqrt{a_{i_{j}}}} a_{i_{j}}+\frac{\sqrt{d_{i_{k}}}+\sqrt{a_{i_{k}}}}{\Delta \sqrt{d_{i_{k}}}} d_{i_{k}} \\
& \leq \sum_{j=1}^{k-1} \frac{\sqrt{a_{i_{0}}}+\sqrt{d_{i_{0}}}}{\Delta \sqrt{d_{i_{0}}}} a_{i_{j}}+\frac{\sqrt{d_{i_{0}}}+\sqrt{a_{i_{0}}}}{\Delta \sqrt{d_{i_{0}}}} d_{i_{k}}=\frac{\sqrt{a_{i_{0}}}+\sqrt{d_{i_{0}}}}{\Delta \sqrt{d_{i_{0}}}} \delta^{S}=l_{+} \delta^{S},
\end{aligned}
$$

where the inequality follows from the fact that $\frac{\sqrt{a}+\sqrt{a-\Delta}}{\sqrt{a-\Delta}}=1+\sqrt{1+\frac{\Delta}{a-\Delta}}$ is decreasing on $a$ for $a>\Delta$, and the second last equality holds because $\delta^{S}=\sum_{j=1}^{k-1} a_{i_{j}}+d_{i_{k}}$.

On the other hand, if $a_{i_{k}}=\Delta$, then we have $d_{i_{k}}=d_{i_{j}}=0$ for any $i_{j} \in S$. Thus

$$
\phi\left(\delta^{S}\right)=\sum_{j=1}^{k-1} \frac{a_{i_{j}}+\sqrt{a_{i_{j}} d_{i_{j}}}}{\Delta}+\frac{d_{i_{k}}+\sqrt{a_{i_{k}} d_{i_{k}}}}{\Delta}=\frac{1}{\Delta} \delta^{S} \leq l_{+} \delta^{S} .
$$

Next, we derive an over-approximation of $\phi(\delta)$ when $\delta \leq 0$.

Lemma 8. Define $l_{-}:=\frac{1}{\Delta}$. For $\delta \leq 0$, we have

$$
\phi(\delta)=\left\{\begin{array}{rr}
-\infty & \delta<-\Delta \\
\frac{\sqrt{a_{n}-\Delta}-\sqrt{a_{n}-\Delta-\delta}}{\sqrt{a_{n}}-\sqrt{a_{n}-\Delta}} & -\Delta \leq \delta \leq 0 .
\end{array}\right.
$$

Further, $\phi(\delta) \leq l_{-} \delta$ for $\delta \in[-\Delta, 0]$.

Proof. When $\delta<-\Delta, \phi(\delta)=-\infty$ as the right-hand-side of the problem defining $\phi$ is larger than $\sum_{i=1}^{n} a_{i}$. Consider therefore the case when $0 \geq \delta \geq-\Delta$. There exists an optimal solution $\left(x^{*}, y^{*}\right)$ of the problem defining $\phi(\delta)$ that is such that $x_{i}^{*}=y_{i}^{*}=1$ for all $i \in[n] \backslash\{j\}$ for some $j \in[n]$. Further, $a_{j} x_{j}^{*} y_{j}^{*}=a_{j}-\Delta-\delta$. We obtain

$$
\phi(\delta)=\max _{j}\left[\frac{\sqrt{a_{j}}-\sqrt{a_{j}-\Delta-\delta}}{\sqrt{a_{j}}-\sqrt{a_{j}-\Delta}}\right]-1=\frac{\sqrt{a_{n}}-\sqrt{a_{n}-\Delta-\delta}}{\sqrt{a_{n}}-\sqrt{a_{n}-\Delta}}-1,
$$

where the last step follows from Lemma 5. Finally, observe that $\phi(\delta)$ is convex in $\delta$. Therefore, by taking a linear inequality tight at $\delta=0$ and $\delta=-\Delta$, we obtain that $\phi(\delta) \leq \delta / \Delta=l_{-} \delta$ since $\phi(0)=0$ and $\phi(-\Delta)=-1$. 
By combining Lemmas 7 and 8, we obtain the following over-approximation of $\phi$ :

$$
\phi(\delta) \leq \tilde{\psi}(\delta):=\left\{\begin{array}{rr}
-\infty & \\
l_{-} \delta & -\Delta \leq \delta \leq-\Delta \\
l_{+} \delta & 0 \leq \delta .
\end{array}\right.
$$

Note that the function $\tilde{\psi}$ is not subadditive. Lemma 9 describes a subadditive function that upper bounds $\tilde{\psi}$, thus giving a subadditive upper bound of $\phi$.

Lemma 9. It holds that $l_{+} \geq l_{-}>0$. Further, the function

$$
\psi(\delta)=\left\{\begin{array}{lr}
l_{+}(\delta+\Delta)-l_{-} \Delta & \delta \leq-\Delta \\
l_{-} \delta & -\Delta \leq \delta \leq 0 \\
l_{+} \delta & 0 \leq \delta
\end{array}\right.
$$

is subadditive.

Proof. Define $\dot{\psi}(\delta):=l_{-} \delta$ when $\delta \leq 0$ and $\dot{\psi}(\delta):=l_{+} \delta$ when $\delta \geq 0$. Function $\dot{\psi}(\delta)$ satisfies $\dot{\psi} \geq \psi$ and is subadditive since it is straightforward to verify that $l_{+} \geq l_{-}>0$. Thus, for $u, v$, such that $u, v, u+v \in[-\Delta,+\infty)$, we already have that $\psi(u)+\psi(v) \geq \psi(u+v)$. It remains to consider the cases where at least one of $u, v$ or $u+v$ belongs to $(-\infty,-\Delta]$. We do so by considering the possible values of $u+v$ and by assuming without loss of generality that $u \geq v$. We use the fact that for $\delta \leq 0, \psi(\delta)=\min \left\{l_{+}(\delta+\Delta)-l_{-} \Delta, l_{-} \delta\right\} \geq l_{+} \delta$. There are three cases to consider. First assume that $u+v \geq 0$. In this case, $\psi(u)+\psi(v)-\psi(u+v) \geq l_{+} u+l_{+} v-l_{+}(u+v)=0$. Second assume that $-\Delta \leq u+v \leq 0$. In this case, $v \leq-\Delta$ and $u \geq 0$ so that $\psi(u)+\psi(v)-\psi(u+v)=$ $l_{+} u+l_{+}(v+\Delta)-l_{-} \Delta-l_{-}(u+v)=\left(l_{+}-l_{-}\right)(u+v+\Delta) \geq 0$. Third assume that $u+v \leq-\Delta$. There are two subcases. If $v \leq-\Delta$, we have $\psi(u)+(\psi(v)-\psi(u+v)) \geq l_{+} u+\left(l_{+}(v+\Delta)-l_{+}(u+v+\Delta)\right)=0$. If $v \geq-\Delta$, then $0 \geq u \geq v \geq-\Delta$. Therefore $\psi(u)+\psi(v)-\psi(u+v) \geq l_{-} u+l_{-} v-l_{-}(u+v)=0$.

Theorem 5. Combining Lemmas 7 , 8 , and 9 yields Theorem 5 .

\section{Proof of Theorem 6}

Theorem [6. Following Theorems 3 and 5 , it is sufficient to show that $\gamma_{i}(x, y) \geq \psi\left(a_{i} x y\right)$ for $i \in J_{0}$ and $\gamma_{i}(x, y) \geq$ $\psi\left(a_{i}(x y-1)\right)$ for $i \in J_{1}$, where $\psi$ is the subadditive over-approximation of $\phi$ derived in Theorem 5 . We discuss the possible cases.

(i) Assume $i \in J_{0}^{+}$. We must find $\gamma_{i}(x, y) \geq \psi\left(a_{i} x y\right)=l_{+} a_{i} x y$ for $(x, y) \in[0,1]^{2}$ where the equality holds as $a_{i}>0$. As $\min \{x, y\} \geq x y$ is the best concave upper bound for $(x, y) \in[0,1]^{2}$, we choose $\gamma_{i}(x, y)=l_{+} a_{i} \min \{x, y\}$.

(ii) Assume $i \in J_{1}^{-}$. We must find $\gamma_{i}(x, y) \geq \psi\left(a_{i}(x y-1)\right)=l_{+}\left(a_{i} x y-a_{i}\right)=l_{+} a_{i}(x y-1)$ for $(x, y) \in[0,1]^{2}$ where the equality holds since $a_{i}<0$. As $\max \{x+y-1,0\} \leq x y$ is the best convex lower bound for $(x, y) \in[0,1]^{2}$, we choose $\gamma_{i}(x, y)=l_{+} a_{i}(\max \{x+y-1,0\}-1)=-l_{+} a_{i} \min \{2-x-y, 1\}$.

(iii) Assume $i \in J_{0}^{-}$. We must find $\gamma_{i}(x, y) \geq \psi\left(a_{i} x y\right)$ for $(x, y) \in[0,1]^{2}$. As $a_{i}<0, \psi\left(a_{i} x y\right)=\min \left\{l_{-} a_{i} x y, l_{+} a_{i} x y+\right.$ $\left.l_{+} \Delta-1\right\} \leq \min \left\{l_{-} a_{i}(x+y-1), l_{+} a_{i}(x+y-1)+l_{+} \Delta-1,0\right\}:=\gamma_{i}(x, y)$.

(iv) Assume $i \in J_{1}^{+}$. In this case, we must find $\gamma_{i}(x, y) \geq \psi\left(a_{i} x y-a_{i}\right)$ for $(x, y) \in[0,1]^{2}$. Since $a_{i}>0$, $\psi\left(a_{i} x y-a_{i}\right)=\min \left\{l_{-} a_{i}(x y-1), l_{+} a_{i}(x y-1)+l_{+} \Delta-1\right\}$. Similar to (iii), we have $\psi\left(a_{i} x y-a_{i}\right) \leq$ $l_{-} a_{i}(\min \{x, y\}-1)=: \tilde{h}(x, y)$, and $\psi\left(a_{i} x y-a_{i}\right) \leq l_{+} a_{i}(\min \{x, y\}-1)+l_{+} \Delta-1=: \tilde{g}(x, y)$. Thus, $\gamma(x, y)=\min \{\tilde{h}(x, y), \tilde{g}(x, y)\}$ is a concave upper bound of $\psi$.

Next we improve this upper bound when $a_{i} \geq a_{i_{0}}>\Delta$. As $g$ and $h$ (defined in Theorem 6) are concave, it remains to show the following:

Claim 1. For $a_{i} \geq a_{i_{0}}>\Delta, \min \{g(x, y), h(x, y)\} \geq \psi\left(a_{i} x y-a_{i}\right)$ for $(x, y) \in[0,1]^{2}$. 
Observe that

$$
\psi\left(a_{i} x y-a_{i}\right)=\left\{\begin{array}{lll}
l_{+} a_{i}\left((\sqrt{x y})^{2}-1\right)+l_{+} \Delta-1 & \text { if } & 0 \leq \sqrt{x y} \leq \sqrt{1-\frac{\Delta}{a_{i}}} \\
l_{-} a_{i}\left((\sqrt{x y})^{2}-1\right) & \text { if } & \sqrt{1-\frac{\Delta}{a_{i}}} \leq \sqrt{x y} \leq 1 .
\end{array}\right.
$$

Consider first the function $g_{i}(x, y)=\sqrt{a_{i}-\Delta} \sqrt{a_{i}} l_{+} \sqrt{x y}-l_{+}\left(a_{i}-\Delta\right)-1$ :

- $\sqrt{x y} \in\left[0, \sqrt{1-\frac{\Delta}{a_{i}}}\right]: g_{i}(x, y) \geq-1+\left(a_{i}(\sqrt{x y})^{2}-a_{i}+\Delta\right) l_{+}=\psi\left(a_{i} x y-a_{i}\right)$.

- $\sqrt{x y} \in\left[\sqrt{1-\frac{\Delta}{a_{i}}}, 1\right]$ : we simply prove $\hat{g}_{i}(t):=l_{+} \sqrt{a_{i}-\Delta} \sqrt{a_{i}} \sqrt{t}-l_{+}\left(a_{i}-\Delta\right)-1 \geq l_{-} a_{i}(t-1)=: f_{i}(t)$, for $t \in\left[1-\Delta / a_{i}, 1\right]$. To this end, we verify: (i) $\hat{g}_{i}\left(1-\frac{\Delta}{a_{i}}\right)=f_{i}\left(1-\frac{\Delta}{a_{i}}\right)$ and (ii) $\hat{g}_{i}(1) \geq f_{i}(1)$. This is sufficient since $\hat{g}_{i}$ is a concave function and $f_{i}$ is a linear function. The proof of (i) is straightforward. To prove (ii) observe that $\hat{g}_{i}(1)=l_{+} \sqrt{a_{i}-\Delta} \sqrt{a_{i}}-l_{+}\left(a_{i}-\Delta\right)-1 \geq f_{i}(1)=l_{-} a_{i}(1-1)=0$ is equivalent to verifying $l_{+} \geq \frac{1}{\sqrt{a_{i}-\Delta}\left(\sqrt{a_{i}}-\sqrt{a_{i}-\Delta}\right)}$ or equivalently $\frac{\sqrt{a_{i_{0}}}+\sqrt{a_{i_{0}}-\Delta}}{\sqrt{a_{i_{0}}-\Delta}} \geq \frac{\sqrt{a_{i}}+\sqrt{a_{i}-\Delta}}{\sqrt{a_{i}-\Delta}}$ which holds since $a_{i} \geq a_{i_{0}}$.

Consider second the function $h_{i}(x, y)=\frac{\sqrt{a_{i}}}{\sqrt{a_{i}}-\sqrt{d_{i}}}(\sqrt{x y}-1)$ :

- $\sqrt{x y} \in\left[\sqrt{1-\frac{\Delta}{a_{i}}}, 1\right]$ : by construction, $h_{i}(x, y) \geq l_{-} a_{i}(x y-1) \geq \psi\left(a_{i} x y-a_{i}\right)$.

- $\sqrt{x y} \in\left[0, \sqrt{1-\frac{\Delta}{a_{i}}}\right]$ : first observe that $\hat{h}_{i}\left(t^{*}\right)=f_{i}\left(t^{*}\right)$ for $t^{*}=\sqrt{1-\frac{\Delta}{a_{i}}}$, where $f_{i}(t):=l_{+} a_{i}(t-1)+$ $l_{+} \Delta-1$. Since $h_{i}(x, y)$ is concave it is sufficient to verify that $\hat{h}_{i}(0) \geq f_{i}(0)$. This condition holds as $\frac{\sqrt{a_{i}}}{\sqrt{a_{i}}-\sqrt{a_{i}-\Delta}} \leq 1+\left(a_{i}-\Delta\right) l_{+}$which is equivalent to $l_{+} \geq \frac{1}{\sqrt{a_{i}-\Delta}\left(\sqrt{a_{i}}-\sqrt{a_{i}-\Delta}\right)}$.

\section{References}

[1] Agostinho Agra and Miguel Fragoso Constantino. Lifting two-integer knapsack inequalities. Math. Program. , 109(1):115-154, 2007.

[2] Kurt M. Anstreicher, Samuel Burer, and Kyungchan Park. Convex hull representations for bounded products of variables. arXiv preprint arXiv:2004.07233, 2020.

[3] Alper Atamtürk. On the facets of the mixed-integer knapsack polyhedron. Math. Program., 98(1):145-175, 2003.

[4] Alper Atamtürk. Sequence independent lifting for mixed-integer programming. Oper. Res. , 52(3):487-490, 2004.

[5] Alper Atamtürk and Vishnu Narayanan. Lifting for conic mixed-integer programming. Math. Program. , 126(2):351-363, 2011.

[6] Gennadiy Averkov and Amitabh Basu. Lifting properties of maximal lattice-free polyhedra. Math. Program. , 154(1-2):81-111, 2015.

[7] Egon Balas. Facets of the knapsack polytope. Math. Program. , 8(1):146-164, 1975.

[8] Egon Balas. Disjunctive programming: Properties of the convex hull of feasible points. Discrete Appl. Math. , 89(1-3):3-44, 1998.

[9] Egon Balas and Robert G. Jeroslow. Strengthening cuts for mixed integer programs. Eur. J. Oper. Res. , $4(4): 224-234,1980$. 
[10] Egon Balas and Eitan Zemel. Facets of the knapsack polytope from minimal covers. SIAM J. Appl. Math. , 34(1):119-148, 1978.

[11] Amitabh Basu, Manoel Campêlo, Michele Conforti, Gérard Cornuéjols, and Giacomo Zambelli. Unique lifting of integer variables in minimal inequalities. Math. Program. , 141(1-2):561-576, 2013.

[12] Amitabh Basu, Gérard Cornuéjols, and Matthias Köppe. Unique minimal liftings for simplicial polytopes. Math. Oper. Res. , 37(2):346-355, 2012.

[13] Amitabh Basu, Santanu S. Dey, and Joseph Paat. Nonunique lifting of integer variables in minimal inequalities. SIAM J. Discrete Math., 33(2):755-783, 2019.

[14] Amitabh Basu and Joseph Paat. Operations that preserve the covering property of the lifting region. SIAM J. Optim. , 25(4):2313-2333, 2015.

[15] Aharon Ben-Tal and Arkadi Nemirovski. Lectures on modern convex optimization: analysis, algorithms, and engineering applications. SIAM, 2001.

[16] Daniel Bienstock, Chen Chen, and Gonzalo Munoz. Outer-product-free sets for polynomial optimization and oracle-based cuts. Math. Program. , 183:1-44, 2020.

[17] Merve Bodur, Alberto Del Pia, Santanu S Dey, Marco Molinaro, and Sebastian Pokutta. Aggregation-based cutting-planes for packing and covering integer programs. Math. Program. , 171(1):331-359, 2018.

[18] Samuel Burer. A gentle, geometric introduction to copositive optimization. Math. Program. , 151(1):89-116, 2015.

[19] Sebastián Ceria, Cécile Cordier, Hugues Marchand, and Laurence A. Wolsey. Cutting planes for integer programs with general integer variables. Math. Program., 81(2):201-214, 1998.

[20] Kwanghun Chung, Jean-Philippe P. Richard, and Mohit Tawarmalani. Lifted inequalities for 0-1 mixedinteger bilinear covering sets. Math. Program., 145(1-2):403-450, 2014.

[21] Michele Conforti, Gérard Cornuéjols, and Giacomo Zambelli. A geometric perspective on lifting. Oper. Res. , 59(3):569-577, 2011.

[22] Santanu S. Dey and Jean-Philippe P. Richard. Linear-programming-based lifting and its application to primal cutting-plane algorithms. INFORMS J. Comput. , 21(1):137-150, 2009.

[23] Santanu S. Dey, Asteroide Santana, and Yang Wang. New SOCP relaxation and branching rule for bipartite bilinear programs. Optim. Eng. , 20(2):307-336, 2019.

[24] Santanu S. Dey and Laurence A. Wolsey. Composite lifting of group inequalities and an application to two-row mixing inequalities. Discrete Optim. , 7(4):256-268, 2010.

[25] Santanu S. Dey and Laurence A. Wolsey. Constrained infinite group relaxations of MIPs. SIAM J. Optim. , 20(6):2890-2912, 2010.

[26] Santanu S. Dey and Laurence A. Wolsey. Two row mixed-integer cuts via lifting. Math. Program. , 124(12):143-174, 2010.

[27] Daniel Espinoza, Ricardo Fukasawa, and Marcos Goycoolea. Lifting, tilting and fractional programming revisited. Oper. Res. Lett. , 38(6):559-563, 2010.

[28] Andres Gómez. Submodularity and valid inequalities in nonlinear optimization with indicator variables, 2018. Available at Optimization online.

[29] Ralph E. Gomory and Ellis L. Johnson. Some continuous functions related to corner polyhedra. Math. Program. , 3(1):23-85, 1972. 
[30] Xiaoyi Gu, Santanu S. Dey, and Jean-Philippe P. Richard. Lifting convex inequalities for bipartite bilinear programs. In Mohit Singh and David P. Williamson, editors, Integer Programming and Combinatorial Optimization - 22nd International Conference, IPCO 2021, Atlanta, GA, USA, May 19-21, 2021, Proceedings, volume 12707 of Lecture Notes in Computer Science, pages 148-162. Springer, 2021.

[31] Zonghao Gu, George L. Nemhauser, and Martin W. P. Savelsbergh. Lifted flow cover inequalities for mixed 0-1 integer programs. Math. Program., 85(3):439-467, 1999.

[32] Zonghao Gu, George L. Nemhauser, and Martin W. P. Savelsbergh. Sequence independent lifting in mixed integer programming. J. Comb. Optim. , 4(1):109-129, 2000.

[33] Oktay Günlük and Yves Pochet. Mixing mixed-integer inequalities. Math. Program., 90(3):429-457, 2001.

[34] Akshay Gupte. Mixed integer bilinear programming with applications to the pooling problem. PhD thesis, Georgia Institute of Technology, 2012.

[35] Peter L. Hammer, Ellis L. Johnson, and Uri N. Peled. Facet of regular 0-1 polytopes. Math. Program. , 8(1):179-206, 1975.

[36] Konstantinos Kaparis and Adam N. Letchford. Local and global lifted cover inequalities for the 0-1 multidimensional knapsack problem. Eur. J. Oper. Res. , 186(1):91-103, 2008.

[37] Matthias Köppe and Yuan Zhou. An electronic compendium of extreme functions for the Gomory-Johnson infinite group problem. Oper. Res. Lett. , 43(4):438-444, 2015.

[38] Alexander Martin and Robert Weismantel. The intersection of knapsack polyhedra and extensions. In International Conference on Integer Programming and Combinatorial Optimization, pages 243-256. Springer, 1998.

[39] Garth P. McCormick. Computability of global solutions to factorable nonconvex programs: Part i - convex underestimating problems. Math. Program., 10(1):147-175, 1976.

[40] Gonzalo Muñoz and Felipe Serrano. Maximal quadratic-free sets. In International Conference on Integer Programming and Combinatorial Optimization, pages 307-321. Springer, 2020.

[41] Amar K. Narisetty, Jean-Philippe P. Richard, and George L. Nemhauser. Lifted tableaux inequalities for 0-1 mixed-integer programs: A computational study. INFORMS J. Comput. , 23(3):416-424, 2011.

[42] Trang T. Nguyen, Jean-Philippe P. Richard, and Mohit Tawarmalani. Deriving convex hulls through lifting and projection. Math. Program., 169(2):377-415, 2018.

[43] Manfred W. Padberg. On the facial structure of set packing polyhedra. Math. Program. , 5(1):199-215, 1973.

[44] Manfred W. Padberg. A note on zero-one programming. Oper. Res., 23(4):833-837, 1975.

[45] Hamidur Rahman and Ashutosh Mahajan. Facets of a mixed-integer bilinear covering set with bounds on variables. J. Global Optim., 74(3):417-442, 2019.

[46] Jean-Philippe P. Richard. Lifting techniques for mixed integer programming. Wiley Encyclopedia of Operations Research and Management Science, 2010.

[47] Jean-Philippe P. Richard, Ismael R. de Farias Jr, and George L. Nemhauser. Lifted inequalities for 0-1 mixed integer programming: Basic theory and algorithms. Math. Program. , 98(1-3):89-113, 2003.

[48] Jean-Philippe P. Richard, Ismael R. de Farias Jr, and George L. Nemhauser. Lifted inequalities for 0-1 mixed integer programming: Superlinear lifting. Math. Program., 98(1-3):115-143, 2003.

[49] Jean-Philippe P. Richard and Santanu S. Dey. The group-theoretic approach in mixed integer programming. In 50 Years of Integer Programming 1958-2008, pages 727-801. Springer, 2010. 
[50] Jean-Philippe P. Richard, Yanjun Li, and Lisa A. Miller. Valid inequalities for MIPs and group polyhedra from approximate liftings. Math. Program. , 118(2):253-277, 2009.

[51] Jean-Philippe P. Richard and Mohit Tawarmalani. Lifting inequalities: a framework for generating strong cuts for nonlinear programs. Math. Program., 121(1):61-104, 2010.

[52] Asteroide Santana and Santanu S. Dey. The convex hull of a quadratic constraint over a polytope. SIAM J. Optim. , 30(4):2983-2997, 2020.

[53] Mohit Tawarmalani, Jean-Philippe P. Richard, and Kwanghun Chung. Strong valid inequalities for orthogonal disjunctions and bilinear covering sets. Math. Program. , 124(1-2):481-512, 2010.

[54] Laurence A. Wolsey. Facets and strong valid inequalities for integer programs. Oper. Res. , 24(2):367-372, 1976.

[55] Laurence A. Wolsey. Valid inequalities and superadditivity for 0-1 integer programs. Math. Oper. Res. , $2(1): 66-77,1977$.

[56] Bo Zeng and Jean-Philippe P. Richard. A framework to derive multidimensional superadditive lifting functions and its applications. In International Conference on Integer Programming and Combinatorial Optimization, pages 210-224. Springer, 2007.

[57] Bo Zeng and Jean-Philippe P. Richard. A polyhedral study on 0-1 knapsack problems with disjoint cardinality constraints: facet-defining inequalities by sequential lifting. Discrete Optim. , 8(2):277-301, 2011.

[58] Bo Zeng and Jean-Philippe P. Richard. A polyhedral study on 0-1 knapsack problems with disjoint cardinality constraints: strong valid inequalities by sequence-independent lifting. Discrete Optim. , 8(2):259-276, 2011. 